\title{
A modular metabolic engineering approach for the production of 1,2-propanediol from glycerol by Saccharomyces cerevisiae
}

Islam, Zia-UI; Klein, Mathias; Aßkamp, Maximilian R.; Ødum, Anders Sebastian Rosenkrans; Nevoigt, Elke

Published in:

Metabolic Engineering

Link to article, DOI:

10.1016/j.ymben.2017.10.002

Publication date:

2017

Document Version

Peer reviewed version

Link back to DTU Orbit

Citation $(A P A)$ :

Islam, Z-U., Klein, M., Aßkamp, M. R., Ødum, A. S. R., \& Nevoigt, E. (2017). A modular metabolic engineering approach for the production of 1,2-propanediol from glycerol by Saccharomyces cerevisiae. Metabolic Engineering, 44, 223-235. https://doi.org/10.1016/j.ymben.2017.10.002

\section{General rights}

Copyright and moral rights for the publications made accessible in the public portal are retained by the authors and/or other copyright owners and it is a condition of accessing publications that users recognise and abide by the legal requirements associated with these rights.

- Users may download and print one copy of any publication from the public portal for the purpose of private study or research.

- You may not further distribute the material or use it for any profit-making activity or commercial gain

- You may freely distribute the URL identifying the publication in the public portal 


\section{ACCEPTED MANUSCRIPT}

A modular metabolic engineering approach for the production of

\section{1,2-propanediol from glycerol by Saccharomyces cerevisiae}

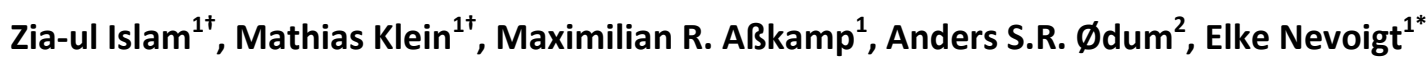

${ }^{1}$ Department of Life Sciences and Chemistry, Jacobs University Bremen gGmbH, Campus Ring 1, 28759 Bremen, Germany

${ }^{2}$ Department of Systems Biology, Building 223, Søltofts Plads, Technical University of Denmark, Lyngby 2800 Kgs, Denmark

${ }^{+}$Equal contributors

* Corresponding author: Mailing address: Jacobs University Bremen gGmbH, Department of Life Sciences and Chemistry, Campus Ring 1, 28759 Bremen, Germany; Phone: +49-421-2003541; Fax: +49-421-2003249; E-mail: e.nevoigt@jacobs-university.de

\section{KEYWORDS}

Yeast, Saccharomyces cerevisiae, glycerol, 1,2-propanediol, dihydroxyacetone, triosephosphate isomerase

\section{RUNNING TITLE}

1,2-propanediol production from glycerol in Saccharomyces cerevisiae 


\section{ACCEPTED MANUSCRIPT}

\section{ABSTRACT}

Compared to sugars, a major advantage of using glycerol as a feedstock for industrial bioprocesses is the fact that this molecule is more reduced than sugars. A compound whose biotechnological production might greatly profit from the substrate's higher reducing power is 1,2-propanediol (1,2PDO). Here we present a novel metabolic engineering approach to produce 1,2-PDO from glycerol in S. cerevisiae. Apart from implementing the heterologous methylglyoxal (MG) pathway for 1,2-PDO formation from dihydroxyacetone phosphate (DHAP) and expressing a heterologous glycerol facilitator, the employed genetic modifications included the replacement of the native FADdependent glycerol catabolic pathway by the 'DHA pathway' for delivery of cytosolic NADH and the reduction of triosephosphate isomerase (TPI) activity for increased precursor (DHAP) supply. The choice of the medium had a crucial impact on both the strength of the metabolic switch towards fermentation in general (as indicated by the production of ethanol and 1,2-PDO) and on the ratio at which these two fermentation products were formed. For example, virtually no 1,2-PDO but only ethanol was formed in synthetic glycerol medium with urea as the nitrogen source. When nutrientlimited complex YG medium was used, significant amounts of 1,2-PDO were formed and it became obvious that the concerted supply of NADH and DHAP are essential for boosting 1,2-PDO production. Additionally, optimizing the flux into the MG pathway improved 1,2-PDO formation at the expense of ethanol. Cultivation of the best-performing strain in YG medium and a controlled bioreactor set-up resulted in a maximum titer of $>4 \mathrm{~g} \mathrm{~L}^{-1} 1,2$-PDO which, to the best of our knowledge, has been the highest titer of 1,2-PDO obtained in yeast so far. Surprisingly, significant 1,2-PDO production was also obtained in synthetic glycerol medium after changing the nitrogen source towards ammonium sulfate and adding a buffer. 


\section{ACCEPTED MANUSCRIPT}

\section{INTRODUCTION}

A major challenge for the production of chemicals by industrial biotechnology is the fact that the commonly used sugar-based substrates inevitably result in low carbon yields which can be attributed to the high oxidation state (O:C ratio) of sugars relative to many products (Burk and Van Dien, 2016). Although glycerol is still less reduced than hydrocarbons derived from fossil resources, the degree of reduction (4.667) is higher than that of common sugars such as glucose (4.000) and xylose (4.000) (Yazdani and Gonzalez, 2008). Therefore, the utilization of glycerol as a substrate or co-substrate in the fermentative production of chemicals can lead to higher maximum theoretical product yields of compounds with a relatively high degree of reduction as well as of molecules whose biochemical production from sugars is not redox-neutral but requires additional reducing equivalents such as 1,2propandiol or succinic acid (Murarka et al., 2008; Yazdani and Gonzalez, 2007; Zhang et al., 2013).

Glycerol is an unavoidable by-product of the biodiesel industry since transesterification of vegetable oils or animal fats generates app. 10\% (w/w) of glycerol (Karinen and Krause, 2006). As a result of the growing demand for renewable transportation fuels, the production of biodiesel steadily increased during the last decades accompanied by the accumulation of huge amounts of crude glycerol (Mattam et al., 2013). In principle, this crude glycerol has to be considered as a waste stream since it still contains various residual components from the transesterification process such as methanol, $\mathrm{NaOH}$, carry-over fats/oils as well as low amounts of proteins, minerals and sulfur compounds (Thompson and He, 2006). The utilization of excess crude glycerol as a feedstock for the microbial production of various value-added chemicals in industrial bioprocesses has been considered as a promising approach for its valorization (da Silva et al., 2009; Johnson and Taconi, 2007; Yang et al., 2012).

The yeast Saccharomyces cerevisiae has become a favorite platform organism for the production of various chemical compounds by means of metabolic engineering. In fact, re-routing the carbon flux in this organism allowed the commercial production of advanced biofuels (e.g. isoprenoids, isobutanol), 


\section{ACCEPTED MANUSCRIPT}

bulk chemicals (e.g. succinic acid) as well as pharmaceuticals and neutraceuticals (Borodina and Nielsen, 2014; Hong and Nielsen, 2012; Nevoigt, 2008). Major factors driving the increasing metabolic engineering incentives in S. cerevisiae are its robustness in industrial setups and its easy accessibility for genetic engineering including the tremendous developments with regard to DNA assembly, chromosomal engineering and genome editing as comprehensively reviewed by Chao et al. (2015) and David and Siewers (2015).

Most of the commonly employed industrial and laboratory strains of S. cerevisiae utilize glycerol only in the presence of complex medium supplements such as amino acids and nucleic bases but do not grow in synthetic medium containing glycerol as the sole carbon source (Merico et al., 2011; Swinnen et al., 2013). Therefore, glycerol has been rather neglected as a feedstock for S. cerevisiae-based bioprocesses. Still, certain wild-type S. cerevisiae isolates including the strain CBS 6412 are able to moderately grow in synthetic glycerol medium and a haploid segregant of this strain (CBS 6412-13A) showed a maximum specific growth rate $\left(\mu_{\max }\right)$ of $\sim 0.13 \mathrm{~h}^{-1}$ (Swinnen et al., 2013). The genetic determinants underlying the latter segregant's positive phenotype with regard to growth on glycerol in comparison to the non-growing popular laboratory strain CEN.PK113-1A have been unraveled recently (Swinnen et al., 2016). Moreover, the maximum specific growth rate of CBS 6412-13A in synthetic glycerol medium has been even further improved up to $\sim 0.18 \mathrm{~h}^{-1}$ by the expression of a glycerol facilitator (Klein et al., 2016b). Several facilitators originating from various yeast species that naturally show superior growth in glycerol medium as compared to S.cerevisiae (such as Cyberlindnera jadinii, Yarrowia lipolytica, Pachysolen tannophilus and Komagataella phaffii syn. Pichia pastoris) had a similar positive effect on glycerol utilization.

Wild-type S. cerevisiae strains exclusively utilize the so-called G3P (L-glycerol-3-phosphate) pathway for catabolizing glycerol as recently reviewed by Klein et al. (2016c). This pathway transfers the electrons derived from G3P oxidation directly via $\mathrm{FADH}_{2}$ to the cell's mitochondrial respiratory chain thereby 'squandering' the substrate's aforementioned superior reducing power. In fact, the respective electrons have to be available in the form of cytosolic NADH in order to support the 


\section{ACCEPTED MANUSCRIPT}

formation of reduced fermentation products. Recently, we replaced the endogenous G3P glycerol catabolic pathway by the so-called DHA pathway which transfers the electrons to cytosolic $\mathrm{NAD}^{+}$ thereby delivering NADH for fermentative pathways (Klein et al., 2016a; Nevoigt, 2017). S. cerevisiae strains engineered in this way show similar or even better glycerol utilization as compared to the corresponding wild-type strains offering the possibility to employ the pathway replacement approach for the production of reduced chemicals from glycerol in S. cerevisiae.

1,2-propanediol (1,2-PDO, propylene glycol) is one example for a reduced compound (degree of reduction per Cmol: 5.333), whose biotechnological production may greatly profit from using glycerol as a feedstock. 1,2-PDO is an important commodity chemical applied in food, drug, and cosmetic industries. It is frequently used for the preparation of unsaturated polyester resins, for the production of biodegradable plastics, as a solvent in photographic industries, as an aircraft de-icing fluid and due to its lower toxicity as an environmentally friendly substitute for ethylene glycol in automotive antifreeze (Berrios-Rivera et al., 2003; Niimi et al., 2011; Shelley, 2007). A wide range of bacteria is naturally able to produce $1,2-$ PDO by fermentation, such as Thermoanaerobacterium thermosaccharolyticum (Cameron and Cooney, 1986), Clostridium sphenoides (Tran-Din and Gottschalk, 1985), Bacteroides ruminocola (Turner and Roberton, 1979), Klebsiella pneumoniae (Badia et al., 1985) and E. coli (Boronat and Aguilar, 1979; Boronat and Aguilar, 1981; Gonzalez et al., 2008; Murarka et al., 2008). Interestingly, there has also been a very early report about some aerobic 1,2-PDO production by several non-conventional yeast species (Suzuki and Onishi, 1968) and small amounts have been detected in several industrial S. cerevisiae strains (Dowd et al., 1994). However, one has to note that a full metabolic pathway for the production of 1,2-PDO has never been identified in yeast. In addition, the respective cultivations were performed in undefined media which may contain one or several of the pathway intermediates. These intermediates could have been converted to 1,2-PDO by endogenous, non-specific oxidoreductase activities (Bennett and San, 2001). 


\section{ACCEPTED MANUSCRIPT}

Two main natural biochemical pathways leading to 1,2-PDO production have been discovered in bacteria. A widely spread pathway, which exists for example in E. coli and Salmonella typhimurium, depends on the fermentation of the deoxy hexose sugars L-rhamnose and L-fucose (Badia et al., 1985; Bennett and San, 2001; Boronat and Aguilar, 1981). A second natural route identified in T. thermosaccharolyticum and C. sphenoides converts the glycolytic carbon intermediate dihydroxyacetone phosphate (DHAP) by a methylglyoxal synthase (MGS) to methylglyoxal (MG) followed by two subsequent $\mathrm{NAD}(\mathrm{P}) \mathrm{H}$ dependent reductions catalyzed by aldo-keto reductases (AKRs) to 1,2-PDO (see Figure 1) (Bennett and San, 2001; Cameron and Cooney, 1986; Tran-Din and Gottschalk, 1985). 1,2-PDO formation by wild-type E. coli had been originally assumed to be restricted to the fermentation of the deoxy hexose sugars as mentioned above. However, more recent studies demonstrated that the so-called 'methylglyoxal pathway' is also used in E. coli to anaerobically ferment glycerol where 1,2-PDO production is used as a redox sink in order to cope with the surplus cytosolic NADH derived from biomass formation (Gonzalez et al., 2008; Murarka et al., 2008). As the latter pathway allows for the production of 1,2-PDO from inexpensive sugars or glycerol it has been the preferred route in the context of rational engineering of microorganisms for 1,2-PDO production. So far, the bacterium E. coli has been the mainly employed chassis in numerous engineering studies aiming at the fermentative production of 1,2-PDO using either glucose (Altaras and Cameron, 1999; Altaras and Cameron, 2000; Berrios-Rivera et al., 2003; Cameron et al., 1998; Huang et al., 1999; Jain et al., 2015a; Jain et al., 2015b; Lee et al., 2016) or glycerol (Clomburg and Gonzalez, 2011) as the sole carbon source. The 'methylglyoxal pathway' was also used to produce 1,2-PDO from glucose in engineered cells of Corynebacterium glutamicum (Niimi et al., 2011; Siebert and Wendisch, 2015) and from $\mathrm{CO}_{2}$ by metabolic engineering of the cyanobacterium Synechococcus elongatus (Li and Liao, 2013). Eukaryotic microorganisms previously engineered for 1,2-PDO production include S. cerevisiae (Jeon et al., 2009; Jung et al., 2008; Jung et al., 2011; Lee and DaSilva, 2006) and K. phaffii (P. pastoris) (Barbier et al., 2011). Notably, the highest titers of 1,2-PDO such as 


\section{ACCEPTED MANUSCRIPT}

$5.62 \mathrm{~g} / \mathrm{L}$ for E. coli (Clomburg and Gonzalez, 2011) and $2.19 \mathrm{~g} / \mathrm{L}$ for S. cerevisiae (Jung et al., 2011) were achieved when using glycerol as the carbon source.

Here, we present a novel metabolic engineering study for the production of 1,2-PDO from glycerol in the yeast $S$. cerevisiae. The current work was initiated since we assumed that our previously reported glycerol catabolic pathway replacement (Klein et al., 2016a) might be crucial for providing sufficient amounts of cytosolic NADH for the production of reduced chemicals such as 1,2-PDO. Apart from obtaining the highest 1,2-PDO titer produced in yeast so far, we also got surprising new insights into the physiology of glycerol metabolism in our engineered strains including unexpected effects caused by the medium composition. 


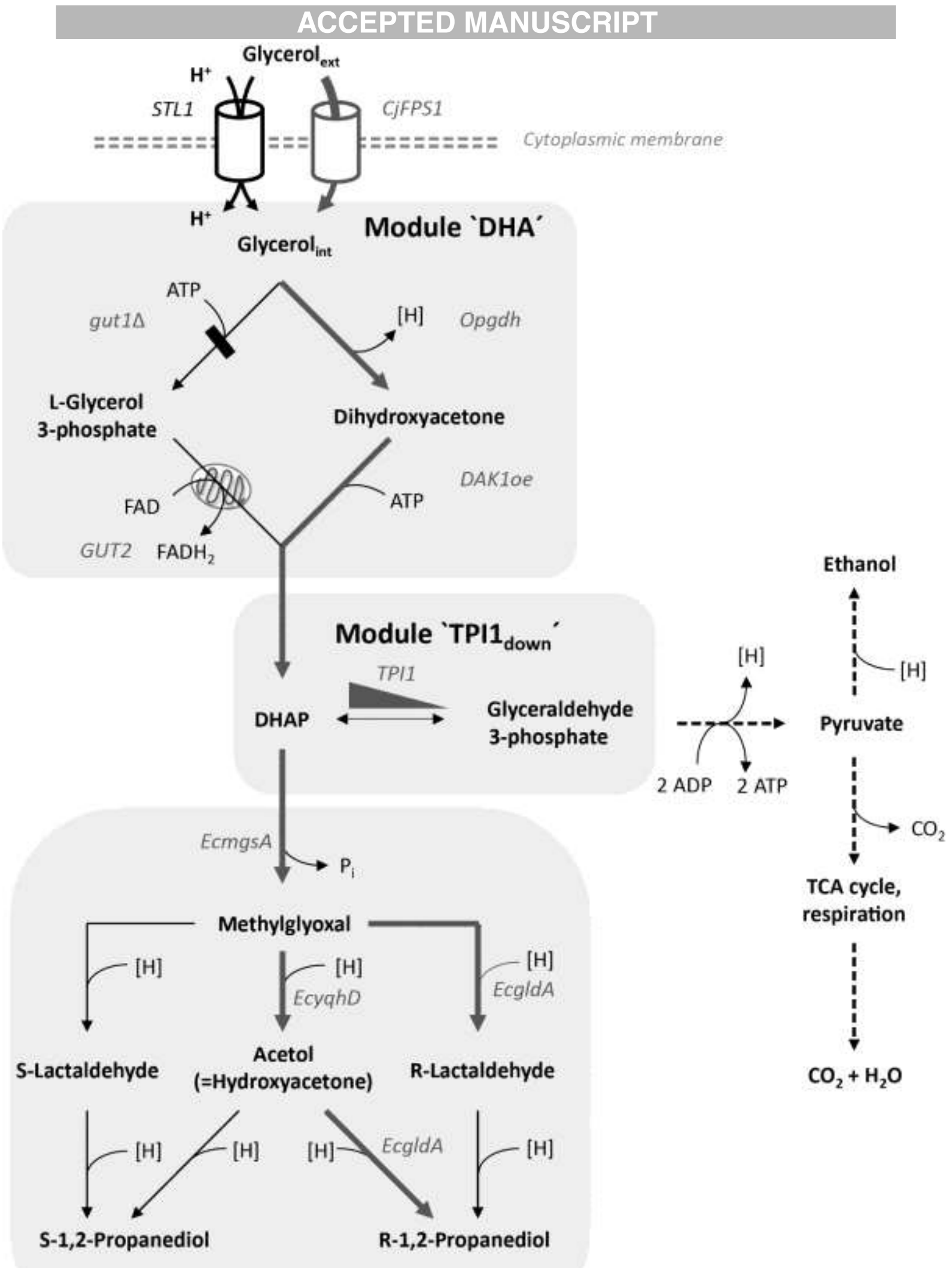

\section{Module 'PDO'}

Figure 1 ( 2 column fitting image). Metabolic engineering for 1,2-propanediol (1,2-PDO) production from glycerol in S. cerevisiae. Module 'PDO': 'methylglyoxal pathway' converting dihydroxyacetone phosphate (DHAP) via methylglyoxal to 1,2-PDO by expressing $E$. coli methylglyoxal synthase (encoded by EcmgsA), E. coli 
aldehyde reductase (encoded by EcyqhD) and E. coli glycerol dehydrogenase (encoded by EcgldA). Module 'DHA': replacement of the native FAD-dependent glycerol catabolic pathway via L-glycerol-3-phosphate by the $\mathrm{NAD}^{+}$-dependent 'DHA pathway' by deletion of the gene encoding glycerol kinase (GUT1), expression of a glycerol dehydrogenase from $O$. parapolymorpha (Opgdh) and overexpression of endogenous dihydroxyacetone kinase (encoded by DAK1). Module 'TPI $1_{\text {down }}$ ': downregulation of the expression of TPI1 encoding triosephosphate isomerase by replacement of the gene's native promoter by the weak TEF1 promoter variant TEFmut2 (Nevoigt et al., 2006). All constructed S. cerevisiae strains express the glycerol facilitator from $C$. jadinii encoded by CjFPS1 in addition to the endogenous glycerol/ $\mathrm{H}^{+}$symporter encoded by STL1. The latter modification has been considered to be useful as it resulted in a significantly improved glycerol uptake in the genetic background of CBS 6412-13A (Klein et al., 2016b). Bold grey arrows highlight the carbon flux from glycerol to 1,2-PDO as it is assumed based on the published knowledge regarding the in vivo and/or in vitro activities of transport and enzymes as explained in the main text. Still, it has to be considered that the heterologous enzymes encoded by EcgldA, EcyqhD and Opgdh have relatively broad substrate specificities and might also contribute to other reduction/oxidation reactions in addition those allocated to the gene names in this Figure. Moreover, unspecific/uncharacterized endogenous enzyme activities in S. cerevisiae might also contribute to the different potential reactions from methylglyoxal to 1,2-PDO.

\section{MATERIALS AND METHODS}

\subsection{Strains and their maintenance}

All S. cerevisiae strains used in this study were based on CBS 6412-13A (Swinnen et al., 2013) and are listed in Table 1 . The yeast strains were routinely grown on solid YPD medium containing $10 \mathrm{~g} \mathrm{~L}^{-1}$ yeast extract, $20 \mathrm{~g} \mathrm{~L}^{-1}$ peptone, $20 \mathrm{~g} \mathrm{~L}^{-1}$ glucose, and $15 \mathrm{~g} \mathrm{~L}^{-1}$ agar. All strains, whose native TPI1 promoter had been replaced by the engineered TEF1 promoter variant TEFmut2, were grown on solid YP glycerol medium (YPG) containing $30 \mathrm{~g} \mathrm{~L}^{-1}$ glycerol as the carbon source. If required, solid medium was supplemented with $\mathrm{G} 418\left(200 \mathrm{mg} \mathrm{L}^{-1}\right)$, phleomycin $\left(20 \mathrm{mg} \mathrm{L}^{-1}\right)$, nourseothricin $\left(100 \mathrm{mg} \mathrm{L}^{-1}\right)$ or hygromycin $\mathrm{B}\left(300 \mathrm{mg} \mathrm{L}^{-1}\right)$. E. coli $\mathrm{DH} 5 \alpha$ was used for plasmid construction and isolation, and cells were routinely grown in lysogeny broth (LB) $\left(10 \mathrm{~g} \mathrm{~L}^{-1}\right.$ peptone, $5 \mathrm{~g} \mathrm{~L}^{-1}$ yeast extract and $10 \mathrm{~g} \mathrm{~L}^{-1} \mathrm{NaCl}, \mathrm{pH}$ 7.0). For selection and maintenance of plasmid containing cells, $100 \mathrm{mg} \mathrm{L}^{-1}$ ampicillin was added.

\subsection{General molecular biology techniques}




\section{ACCEPTED MANUSCRIPT}

All primers used within the present study are listed in Table S1 (supplementary information).

Preparative PCRs for cloning were performed using Phusion ${ }^{\circledR}$ High-Fidelity DNA Polymerase (New

England BioLabs, Frankfurt am Main, Germany) or AccuPrime Pfx DNA polymerase (Thermo Fisher

Scientific, Waltham, MA, USA). PCR conditions were adapted to the guidelines of the respective manufacturer. Restriction enzymes, FastAP alkaline phosphatase and T4 DNA ligase were obtained from Thermo Fisher Scientific and used according to the manufacturer's instructions. PCR products were purified by using the QIAquick PCR Purification Kit (Qiagen, Hilden, Germany) and DNA fragments obtained after restriction were excised and purified using the QIAquick Gel Purification Kit (Qiagen). Plasmids were isolated from E. coli $\mathrm{DH} 5 \alpha$ overnight cultures using the QIAprep Spin Miniprep Kit (Qiagen). Transformation of S. cerevisiae with plasmids as well as with linear DNA fragments for genomic integration was performed according to the lithium acetate method described by Gietz et al. (1995).

Table 1: Strains used in this study.

\begin{tabular}{|c|c|c|c|}
\hline Strain / Modules & Genome modifications & Plasmid & Source or reference \\
\hline CBS $6412-13 A$ & - & - & Swinnen et al. (2013) \\
\hline CBS 6412-13A CjFPS1 & YGLCT3:: TEF1p-CjFPS1-CYC1t & - & Klein et al. (2016b) \\
\hline $\begin{array}{l}\text { CBS } 6412-13 A D A K 1_{O E} \\
\text { gut1 }\end{array}$ & $\begin{array}{l}\text { YPRCD15::ACT1p-DAK1-TPS1t; } \\
\text { gut1::loxP-kanMX4-loxP }\end{array}$ & - & Klein et al. (2016a) \\
\hline FPS & YGLCT3:: TEF1p-CjFPS1-CYC1t & p41ble-E-E & This study \\
\hline PDO-FPS & $\begin{array}{l}\text { YGLCT3::TEF1p-EcmgsA-CYC1t:TDH3p-EcgldA-IDP1t- } \\
\text { PGK1p-CjFPS1-RPL15At }\end{array}$ & $\begin{array}{l}\text { p41ble-E-yqhD } \\
\text { (ACT1p-EcyqhD-TPS1t) }\end{array}$ & This study \\
\hline PDO-FPS-DHA & $\begin{array}{l}\text { YGLCT3::TEF1p-EcmgsA-CYC1t:TDH3p-EcgldA-IDP1t- } \\
\text { PGK1p-CjFPS1-RPL15At; YPRCD15::ACT1p-DAK1- } \\
\text { TPS1t; gut1::loxP-kanMX4-loxP }\end{array}$ & $\begin{array}{l}\text { p41ble-gdh-yqhD } \\
\text { (TEF1p-Opgdh-CYC1t; } \\
\text { ACT1p-EcyqhD-TPS1t) }\end{array}$ & This study \\
\hline PDO-FPS-TPI $1_{\text {down }}$ & $\begin{array}{l}\text { YGLCT3::TEF1p-EcmgsA-CYC1t:TDH3p-EcgldA-IDP1t- } \\
\text { PGK1p-CjFPS1-RPL15At; tpi1p::TEFmut2p-TPI1 }\end{array}$ & $\begin{array}{l}\text { p41ble-E-yqhD } \\
\text { (ACT1p-EcyqhD-TPS1t) }\end{array}$ & This study \\
\hline PDO-FPS-TPI $1_{\text {down }}-$ DHA & $\begin{array}{l}\text { YGLCT3::TEF1p-EcmgsA-CYC1t:TDH3p-EcgldA-IDP1t- } \\
\text { PGK1p-CjFPS1-RPL15At; tpi1p::TEFmut2p-TPI1; } \\
\text { YPRCD15::ACT1p-DAK1-TPS1t; gut1::loxP-kanMX4- } \\
\text { loxP }\end{array}$ & $\begin{array}{l}\text { p41ble-gdh-yqhD } \\
\text { (TEF1p-Opgdh-CYC1t; } \\
\text { ACT1p-EcyqhD-TPS1t) }\end{array}$ & This study \\
\hline $\begin{array}{l}\text { PDO-FPS-TPI } 1_{\text {down }}-\text { DHA } \\
\text { EcmgsA }\end{array}$ & $\begin{array}{l}\text { YGLCT3::TEF1p-EcmgsA-CYC1t:TDH3p-EcgldA-IDP1t- } \\
\text { PGK1p-CjFPS1-RPL15At; tpi1p::TEFmut2p-TPI1; } \\
\text { YPRCD15::ACT1p-DAK1-TPS1t; gut1::loxP-kanMX4- } \\
\text { loxP; YPRCT3::TDH3p-EcmgsA-IDP1t: TEF1p-Opgdh- } \\
\text { CYC1t; YORWA22:: ACT1p-EcyqhD-TPS1t }\end{array}$ & - & This study \\
\hline
\end{tabular}




\section{ACCEPTED MANUSCRIPT}

\subsection{Construction of plasmids and expression cassettes}

All constructed plasmids are listed in Table S2 (supplementary information). Codon-optimized coding sequences for $E$. coli $m g s A, g / d A$ and $y q h D$ were obtained via GeneArt ${ }^{\mathrm{TM}}$ gene synthesis from Thermo Fisher Scientific (Waltham, MA, USA). The plasmids for either the sole expression of E. coli yqhD (EcyqhD) under the control of the ACT1 promoter and the TPS1 terminator or its expression in combination with the O. parapolymorpha gdh (Opgdh), under the control of the TEF1 promoter and the CYC1 terminator, were obtained by using Gibson isothermal assembly (Gibson et al., 2009). The assembly was performed in the previously constructed S. cerevisiae expression vectors p41bleTEF or p41bleTEF-Opgdh (Klein et al., 2016a). The ACT1 promoter and the TPS1 terminator sequences were amplified from genomic DNA isolated from the S. cerevisiae strain S288c using primers 531 and 532, and 533 and 534, respectively (see Table S1). The coding sequence of E. coli yqhD was amplified from pMA-T-yqhD (Table S2) using primers 535 and 536, and the template vector was subsequently removed by digestion with $D p n l$. The assembly reactions contained $15 \mu \mathrm{L}$ of the reagent-enzyme mix, $0.05 \mathrm{pmol}$ of $\mathrm{Kpnl}$-linearized and dephosphorylated p41bleTEF or p41bleTEF-Opgdh and 3-fold molar excess of the inserts (promoter, coding sequence and terminator each $0.15 \mathrm{pmol}$ ) in a final volume of $20 \mu \mathrm{L}$. Reaction mixtures were incubated at $50^{\circ} \mathrm{C}$ for 1 h. E. coli DH5 $\alpha$ cells were transformed with $5 \mu \mathrm{L}$ of the reaction and the resulting vectors named p41ble-E-yqhD and p41ble-gdh-yqhD where "E" specifies an empty expression cassette consisting solely of the TEF1 promoter and CYC1 terminator sequences (Table S2). The empty vector control (carrying two empty expression cassettes) was constructed accordingly by assembling only the ACT1 promoter and the TPS1 terminator in p41bleTEF. The primers used for amplification were 531 and 538, and 533 and 537, respectively. The resulting 'empty' vector was named p41ble-E-E and used for the construction of control strains.

The expression cassettes for C. jadinii FPS1 (CjFPS1) and S. cerevisiae DAK1 (SCDAK1) for genomic integration were obtained by PCR amplification from the plasmids pUC18-CjFPS1 and pUC18-DAK1 (Table S2). Construction of these plasmids is described in Klein et al. (2016a). One of the two expression cassettes constructed for integrating E. coli mgsA into the genome (EcmgsA under the 


\section{ACCEPTED MANUSCRIPT}

control of the S. cerevisiae TEF1 promoter and the CYC1 terminator) was obtained by cloning the respective coding sequence into the multiple cloning site of p416TEF-KanMX (Table S2). For this purpose the EcmgsA coding sequence was amplified from pMA-T-mgs (Table S2) using primers 276 and 277. The cloning via BamHI and EcoRl yielded the plasmid p416TEF-mgsA-KanMX. The second EcmgsA expression cassette $\left(E c m g s A_{2}\right.$ under the control of the TDH3 promoter and the IDP1 terminator) and the cassette for expression of E. coli gldA (EcgldA) (also under the control of the TDH3 promoter and the IDP1 terminator) were both assembled in pUC18 using Gibson isothermal assembly (Gibson et al., 2009). Promoters and terminators were amplified from genomic DNA isolated from the S. cerevisiae strain S288c using primer combinations $413 / 419$ and $415 / 328$ for the expression cassette of $E c m g s A_{2}$, and 324/323 and 327/328 for the expression cassette of EcgldA. The coding sequences for EcmgsA and EcgldA were amplified from the plasmids pMA-T-mgs and pGAgldA using primer combinations $420 / 414$ and $325 / 326$, respectively. Both template vectors were removed after PCR by digestion with DpnI. The target vector pUC18 was linearized using BamHI and subsequently dephosphorylated to avoid self-ligation. The further steps for assembly and transformation were the same as described above and the resulting plasmids named pUC18-gldA and pUC18-mgsA. All constructed plasmids were verified by Sanger sequencing (GATC Biotech, Konstanz, Germany).

\subsection{Plasmids for CRISPR-Cas9 mediated genome editing in S. cerevisiae}

For CRISPR-Cas9 mediated genome editing, the plasmids p414-TEF1p-Cas9-CYC1t-nat1 and p426SNR52p-gRNA.CAN1.Y-SUP4t-hphMX (Table S2) were used. Construction of the respective plasmids has been reported by Klein et al. (2016a). The specific 20 nt sequence at the $5^{\prime}$-end of the gRNA targeting the CAN1 gene was exchanged in p426-SNR52p-gRNA.CAN1.Y-SUP4t-hphMX by a $20 \mathrm{nt}$ sequence targeting the YGLCT3 region on chromosome VII (Flagfeldt et al., 2009) by generating two overlapping PCR products of the gRNA expression cassette and their subsequent assembly in the same vector backbone according to Gibson et al. (2009). The target sequence within the YGLCT3 locus was selected after analysis of the respective genomic region using the CRISPRdirect online tool 


\section{ACCEPTED MANUSCRIPT}

developed by Naito et al. (2015). The two overlapping fragments of the gRNA expression cassette were obtained by PCR amplification from p426-SNR52p-gRNA.CAN1.Y-SUP4t (Table S2) using primers 460 and 464, and 465 and 463, respectively (Table S1), where the $5^{\prime}$-termini of primers 464 and 465 generated flanking sequences at the ends of the two PCR products which overlapped with the YGLC 23 target sequence for genomic integration. The primers 460 and 463 contained 5 '-extensions resulting in overlaps with Pvull-linearized p426-SNR52p-gRNA.CAN1.Y-SUP4t-hphMX. The accordingly digested vector was gel-purified and Gibson isothermal assembly was performed as described above (section 2.3) yielding p426-SNR52p-gRNA.YGLCt3-SUP4t-hphMX.

\subsection{S. cerevisiae strain construction}

A detailed scheme for the construction of engineered CBS 6412-13A derivatives (Table 1) is shown in Figure S1 (supplementary information). As the expression of EcyqhD and Opgdh in all constructed strains except PDO-FPS-TPI $1_{\text {down }}$-DHA EcmgsA $A_{2}$ was plasmid-based, the final step for completion was always a transformation with p41ble-E-yqhD, p41ble-gdh-yqhD or p41ble-E-E.

\section{Strains PDO-FPS and FPS}

As mentioned in section 2.4 the long terminal repeat (LTR) YGLCT3 on chromosome VII (Flagfeldt et al., 2009) was selected as a target site for the genomic integration of the expression cassettes for EcmgsA (under the control of the TEF1 promoter and the CYC1 terminator) and EcgldA (under the control of the TDH3 promoter and the IDP1 terminator) in CBS 6412-13A. The seamless integration was achieved in two steps. First, a counter-selectable, galactose-inducible growth inhibitory sequence (referred to as GALp-GIN11M86) and the kanMX4 selectable marker were assembled and integrated at the target site by homologous recombination. The GALP-GIN11M86 cassette was amplified from plasmid pGG119 (Akada et al., 2002) using primers 175 and 155 . The kanMX4 expression cassette was amplified from plasmid pUG6 (Gueldener et al., 1996) using primers 171 and 176. The PCR primers were modified at their $5^{\prime}$-ends generating homologous regions of $\sim 40-60$ bp at the ends of the PCR products for assembly and integration at the target locus. In the second step, the 


\section{ACCEPTED MANUSCRIPT}

two aforementioned expression cassettes EcmgsA and EcgldA were assembled and integrated by homologous recombination at the target locus replacing the entire GALp-GIN11M86/kanMX4 cassette. The respective expression cassettes were amplified from the previously constructed plasmids p416TEFmgsA-KanMX (primers 257 and 302) and pUC18-gldA (primers 303 and 297), respectively. Primer pairs were designed to generate PCR products whose flanking sequences were homologous to the chromosomal regions upstream and downstream of the previously integrated GALp-GIN11M86/kanMX4 cassette. After transformation, cells were plated on solid synthetic medium containing $2 \%(\mathrm{w} / \mathrm{v})$ galactose as the sole carbon source in order to induce the growth inhibitory sequence GIN11M86 for counter-selection. The resulting intermediate strain was named $\mathrm{PDO}_{\text {min }}$ (Figure S1). Subsequently, an expression cassette for CjFPS1 (under the control of the PGK1 promoter and the RPL15A terminator) was integrated together with a phleomycin resistance marker (ble) downstream of EcmgsA and EcgldA. The CjFPS1 cassette was amplified with primers 390 and 392 from pUC18-CjFPS1, while the ble cassette was amplified from pUG66 (Gueldener et al., 2002) using primers 391 and 393 . The 5'-termini of the employed primers were designed to allow assembly and integration of the generated PCR products into the genome of strain $\mathrm{PDO}_{\min }$, immediately downstream of the previously integrated expression cassettes for EcmgsA and EcgldA, and the resulting strain was named $\mathrm{PDO}_{\min }-\mathrm{FPS}$. The phleomycin resistance cassette was subsequently removed from this intermediate strain by using Cre-loxP-mediated recombination according to Steensma and Ter Linde (2001) using the Cre-recombinase expressing plasmid pNatCre (Table S2). Finally the resulting strain was transformed with p41ble-E-yqhD to yield the strain PDO-FPS. In order to generate a control strain without an implemented pathway for 1,2-PDO production the previously constructed strain CBS 6412-13A CjFPS1 (Klein et al., 2016b) was transformed with the 'empty' vector p41ble-E-E (section 2.3; Table S2).

\section{Strain PDO-FPS-DHA}

In order to combine the modules for the 1,2-PDO pathway and the glycerol catabolic pathway replacement (DHA pathway replacing the endogenous G3P pathway) in an FPS1 expressing strain of 


\section{ACCEPTED MANUSCRIPT}

CBS $6412-13 \mathrm{~A}$, the previously generated strain CBS $6412-13 \mathrm{~A}$ DAK1 $1_{\text {OE }}$ gut1 $\triangle$ was used as a basis

(Table 1) (Klein et al., 2016a). First, the expression cassettes for EcmgsA, EcgldA and CjFPS1 were integrated into the YGLCT3 region of this strain by employing the CRISPR-Cas9 system. For this purpose, the expression of Cas9 in the strain CBS 6412-13A DAK1 OE gut1D was achieved by transformation with the plasmid p414-TEF1p-Cas9-CYC1t-nat1 (Table S2). The three expression cassettes were PCR-amplified from the plasmids p416TEFmgsA-KanMX, pUC18-gldA and pUC18CjFPS1 (see section 2.3 and Table S2) using primers pairs 257/302, 303/552, and 550/551, respectively. These primers contained 5'-extensions generating $40-60$ bp sequences homologous to regions directly upstream and downstream of the YGLCT3 integration locus or to the respective adjacent expression cassette (Figure S1). Co-transformation of the expression cassettes and plasmid p426-SNR52p-gRNA.YGLCT3-SUP4t-hphMX into the S. cerevisiae strain expressing the Cas9 endonuclease resulted in assembly and integration of all three expression cassettes at the target locus. Positive transformants were selected on YPD agar containing both nourseothricin and hygromycin B as described in section 2.1. Both plasmids were subsequently removed from the resulting clone by serial transfers in YPD medium lacking the respective antibiotics. In order to complete both modules $\left(1,2-{ }^{\prime} P D O\right.$ ' and ' $\left.D H A '\right)$, the resulting intermediate strain was transformed with p41ble-gdh-yqhD (see section 2.3) yielding strain PDO-FPS-DHA.

Strains PDO-FPS-TPI1 down and PDO-FPS-TPI $1_{\text {down }}-D H A$

In order to downregulate TPI1 expression, the gene's native promoter was replaced by a promoter replacement cassette containing the weak TEF1 promoter variant TEFmut2p (Nevoigt et al., 2006). The replacement cassette consisting of the kanMX4 selectable marker and the TEFmut2 promoter variant was amplified from the plasmid p416-TEFmut2-yECitrine (Table S2) using primers 426 and 427. The primers contained 5'-extensions of $60 \mathrm{bp}$ homologous to sequences directly upstream and downstream of the endogenous TPI1 promoter. Strain PDO $_{\min }$-FPS (Figure S1) was transformed with the amplified cassette leading to the replacement of the endogenous TPI1 promoter by the engineered promoter variant TEFmut2. Transformants were selected on medium containing glycerol 


\section{ACCEPTED MANUSCRIPT}

as the carbon source. Both dominant markers (the ble cassette downstream of CjFPS1 and the kanMX4 cassette upstream of TPI1) in the intermediate strain $\mathrm{PDO}_{\min }-\mathrm{FPS}-\mathrm{TPI} 1_{\text {down }}$ were subsequently rescued by Cre-loxP mediated recombination as described for strain $\mathrm{PDO}_{\min }$-FPS (see above). The resulting strain was transformed with plasmid p41ble-E-yqhD yielding strain PDO-FPSTPI $1_{\text {down }}$. The previous strain (PDO $\min -$ FPS-TPI $1_{\text {down }}$ after marker removal) also served as the basis for the construction of strain PDO-FPS-TPI $1_{\text {down }}-\mathrm{DHA}$ (Figure S1). First, the cassette for ScDAK1 overexpression (ACT1 promoter and TPS1 terminator) was integrated via the two-step seamless integration procedure employing the counter-selectable, galactose-inducible growth inhibitory sequence GALp-GIN11M86 as described above. The LTR YPRCA15 on chromosome XVI (Flagfeldt et al., 2009) served as a target site and the GALP-GIN11M86 growth inhibitory sequence (amplified from plasmid pGG119 with primers 298 and 155) was first integrated at this position together with the phleomycin resistance marker (amplified from plasmid pUG66 with primers 171 and 299) (Table S2). The SCDAK1 expression cassette was amplified from plasmid pUC18-DAK1 using primers 388 and 389 and subsequently integrated at the target locus by homologous recombination replacing the entire GALp-GIN11M86/ble cassette. Afterwards, the endogenous GUT1 coding sequence was disrupted by integration of the kanMX4 cassette amplified from plasmid pUG6 using primers 111 and 112 yielding the intermediate strain $\mathrm{PDO}_{\min }-\mathrm{FPS}-\mathrm{TPI} 1_{\text {down }} D A K 1_{O E}$ gut1 $\triangle$. Finally, the resulting intermediate strain was transformed with plasmid p41ble-gdh-yqhD to finalize the construction of the strain PDO-FPS-TPI1 down - DHA.

Strain PDO-FPS-TPI1 ${ }_{\text {down }}$-DHA EcmgsA $A_{2}$

In order to implement a second expression cassette for $m g s A$ into the strain and, at the same time, obtain a CBS 6412-13A derivative that carries all above-described genetic modifications for 1,2-PDO production in the genome, the strain PDO-FPS-TPI1 down-DHA EcmgsA $A_{2}$ was generated (Figure S1). The aforementioned intermediate strain $\mathrm{PDO}_{\min }-\mathrm{FPS}-\mathrm{TPI} 1_{\text {down }} D A K 1_{O E}$ gut1 $1 \triangle$ was used as a starting point for the integration of the second expression cassette for $E c m g s A$ (Ecmgs $A_{2}$, coding sequence under the control of the TDH3 promoter and the IDP1 terminator). Similarly to the expression cassettes of 


\section{ACCEPTED MANUSCRIPT}

EcmgsA, EcgldA and ScDAK1, the cassette was integrated together with that of Opgdh into the genome via the two-step seamless integration procedure described above using the LTR YPRCT3 on chromosome XVI as a target site (Flagfeldt et al., 2009). First, the GALp-GIN11M86 growth inhibitory sequence (amplified from plasmid pGG119 with primers 300 and 155) and the phleomycin resistance marker (amplified from plasmid pUG66 with primers 171 and 301) were assembled and integrated at the target locus by homologous recombination. The whole Galp-GIN11M86/ble cassette was subsequently replaced by assembly and integration of the $E c m g s A_{2}$ and $O p g d h$ expression cassettes (amplified with primers 418 and 422 from plasmid pUC18-mgsA and with primers 416 and 421 from plasmid p41bleTEF-Opgdh). Subsequently, the above-described expression cassette for E. coli yqhD was integrated along with the phleomycin resistance marker at the YORW $\Delta 22$ locus (Flagfeldt et al., 2009) yielding strain PDO-FPS-TPI1down-DHA EcmgsA $A_{2}$ (see Figure S1). The EcyqhD cassette was amplified with primers 663 and 664 from plasmid p41ble-E-yqhD, while the ble cassette was amplified from plasmid pUG66 (Gueldener et al., 2002) using primers 665 and 666 . The $5^{\prime}$-termini of the employed primers were designed to allow assembly and integration of the generated PCR products at the target site in the genome.

\subsection{Isolation of genomic DNA from S. cerevisiae transformants and diagnostic PCR}

Correct integrations of all expression and disruption cassettes were verified by diagnostic PCR using TaKaRa Ex Taq Polymerase and buffer according to the manufacturer's guidelines (Merck KGaA, Darmstadt, Germany). For this purpose, single colonies obtained after transformations were restreaked on respective agar plates. Approximately $50 \mathrm{mg}$ of cells from these plates were suspended in $200 \mu \mathrm{L}$ of TE buffer (10 mM Tris, $1 \mathrm{mM}$ EDTA, pH 8.0). Subsequently, $300 \mathrm{mg}$ of acid-washed glass beads (diameter of 0.425 to $0.6 \mathrm{~mm}$ ) and $200 \mu \mathrm{L}$ of phenol:chloroform:isoamyl alcohol (25:24:1) were added. The tubes were then vortexed at maximum speed for 2 min and centrifuged at 15,700 $\mathrm{g}$ for $10 \mathrm{~min}$ according to a protocol modified from Hoffman and Winston (1987). The aqueous phase $(1 \mu \mathrm{L})$ was used as template in $20 \mu \mathrm{L}$ PCR reactions. PCR primers were designed to bind upstream and downstream of the genomic integration sites as well as within the integrated expression/deletion 


\section{ACCEPTED MANUSCRIPT}

cassette. For analyzing integrations of multiple expression cassettes, additional primers were designed to produce amplicons covering the junctions between the individual integrated expression cassettes. After each integration step, the presence of all previously integrated expression cassettes was verified.

\subsection{Media and cultivation conditions for the production of 1,2-PDO from glycerol}

Media used for shake flask batch cultivations assessing 1,2-PDO production were synthetic or complex media containing $60 \mathrm{~mL} \mathrm{~L}^{-1}$ glycerol as the sole carbon source. The synthetic medium according to Verduyn et al. (1992) contained $3 \mathrm{~g} \mathrm{~L}^{-1} \mathrm{KH}_{2} \mathrm{PO}_{4}, 0.5 \mathrm{~g} \mathrm{~L}^{-1} \mathrm{MgSO}_{4} .7 \mathrm{H}_{2} \mathrm{O}, 15 \mathrm{mg} \mathrm{L}^{-1}$ EDTA, $4.5 \mathrm{mg} \mathrm{L}^{-1} \mathrm{ZnSO}_{4} .7 \mathrm{H}_{2} \mathrm{O}, 0.84 \mathrm{mg} \mathrm{L} \mathrm{MnCl}_{2} .2 \mathrm{H}_{2} \mathrm{O}, 0.3 \mathrm{mg} \mathrm{L}^{-1} \mathrm{CoCl}_{2} .6 \mathrm{H}_{2} \mathrm{O}, 0.3 \mathrm{mg} \mathrm{L}{ }^{-1} \mathrm{CuSO}_{4} .5 \mathrm{H}_{2} \mathrm{O}$, $0.4 \mathrm{mg} \mathrm{L}^{-1} \mathrm{NaMoO}_{4} \cdot 2 \mathrm{H}_{2} \mathrm{O}, 4.5 \mathrm{mg} \mathrm{L}^{-1} \mathrm{CaCl}_{2} \cdot 2 \mathrm{H}_{2} \mathrm{O}, 3 \mathrm{mg} \mathrm{L}^{-1} \mathrm{FeSO}_{4} \cdot 7 \mathrm{H}_{2} \mathrm{O}, 1 \mathrm{mg} \mathrm{L}^{-1} \mathrm{H}_{3} \mathrm{BO}_{3}$, and $0.1 \mathrm{mg} \mathrm{L}^{-1} \mathrm{KI}$. In case ammonium sulfate was used as the nitrogen source $5 \mathrm{~g} \mathrm{~L}^{-1}\left(\mathrm{NH}_{4}\right)_{2} \mathrm{SO}_{4}$ was added. After heat sterilization, an appropriate aliquot of vitamin stock solution was added. The final concentrations of vitamins were $0.05 \mathrm{mg} \mathrm{L}^{-1} \mathrm{D}$-(+)-biotin, $1 \mathrm{mg} \mathrm{L}^{-1} \mathrm{D}$-pantothenic acid hemicalcium salt, $1 \mathrm{mg} \mathrm{L}^{-1}$ nicotinic acid, $25 \mathrm{mg} \mathrm{L}^{-1}$ myo-inositol, $1 \mathrm{mg} \mathrm{L}^{-1}$ thiamine chloride hydrochloride, $1 \mathrm{mg} \mathrm{L}^{-1}$ pyridoxine hydrochloride, and $0.2 \mathrm{mg} \mathrm{L}^{-1} 4$-aminobenzoic acid. In case urea was used as the nitrogen source, an appropriate aliquot of a stock solution was added after heat sterilization to obtain a final concentration of $2.8 \mathrm{~g} \mathrm{~L}^{-1}$. For buffering the synthetic medium, $100 \mathrm{mM}$ of potassium hydrogen phthalate was added to the medium prior autoclaving. The $\mathrm{pH}$ of the synthetic glycerol medium was adjusted to 4.0 (for non-buffered medium) and to 5.0 (for medium buffered with potassium hydrogen phthalate) with $2 \mathrm{M} \mathrm{H}_{3} \mathrm{PO}_{4}$. A pH of 4-5 was previously determined to be optimal for the growth on glycerol of wild-type CBS 6412-13A (unpublished results). The complex media used were $\mathrm{YPG}_{6 \%}$ containing $10 \mathrm{~g} \mathrm{~L}^{-1}$ yeast extract, $20 \mathrm{~g} \mathrm{~L}^{-1}$ peptone and $60 \mathrm{~mL} \mathrm{~L}^{-1}$ glycerol or YG containing only $10 \mathrm{~g} \mathrm{~L}^{-1}$ yeast extract in addition to $60 \mathrm{~mL} \mathrm{~L}^{-1}$ glycerol. The $\mathrm{pH}$ of the complex media was adjusted to 6.0 with $2 \mathrm{M} \mathrm{H}_{3} \mathrm{PO}_{4}$. Except for the strain PDO-FPS-TPI $1_{\text {down }}-\mathrm{DHA}-E c m g s A_{2}$, all liquid media were supplemented with $30 \mathrm{mg} \mathrm{L}^{-1}$ phleomycin for plasmid maintenance. 


\section{ACCEPTED MANUSCRIPT}

For pre-cultivation, cells from a single colony were generally used to inoculate $3 \mathrm{~mL}$ of the respective medium in a $10 \mathrm{~mL}$ glass tube and incubated at orbital shaking of $200 \mathrm{rpm}$ and $30^{\circ} \mathrm{C}$ for 24 (complex glycerol medium) to $48 \mathrm{~h}$ (synthetic glycerol medium). The pre-culture was used to inoculate $10 \mathrm{~mL}$ of the same medium in a $100 \mathrm{~mL}$ Erlenmeyer flask adjusting an $\mathrm{OD}_{600}$ of 0.2 . This intermediate culture was cultivated at the same conditions until an $\mathrm{OD}_{600}$ of $\sim 2.0$ was reached. The appropriate culture volume (in order to later adjust an $\mathrm{OD}_{600}$ of 0.2 in $50 \mathrm{~mL}$ of medium) was centrifuged at $800 \mathrm{~g}$ for $5 \mathrm{~min}$ and the supernatant discarded. The cell pellet was then resuspended in $50 \mathrm{~mL}$ of the respective glycerol-containing medium in a $500 \mathrm{~mL}$ Erlenmeyer flask. The main cultures were incubated at orbital shaking of $200 \mathrm{rpm}$ and $30^{\circ} \mathrm{C}$ and samples for $\mathrm{OD}_{600}$ determination and HPLC analysis were taken at regular time intervals.

\subsection{Cultivations in controlled bioreactors}

All reactor-based cultivations were carried out using Sartorious 1 L bioreactors (Sartorious, Stedim Biotech, Göttingen, Germany) with equivalent working volumes and equipped with 2 Rushton sixblade disc turbines. The $\mathrm{pH}$ electrode (Mettler Toledo, $\mathrm{OH}, \mathrm{USA}$ ) was calibrated according to manufacturer's standard procedures and $\mathrm{pH}$ was controlled by automatic addition of $2 \mathrm{M} \mathrm{NaOH}$ and $\mathrm{H}_{2} \mathrm{SO}_{4}$. The bioreactor was sparged with sterile atmospheric air and the temperature was maintained at $30^{\circ} \mathrm{C}$ throughout the cultivations. Culture conditions were: $\mathrm{pH} \mathrm{5}$, stirring rate: $800 \mathrm{rpm}$ and air flow: 1 volume of air per volume of liquid per minute (vvm). Samples were taken across the exponential growth phase to monitor growth $\left(\mathrm{OD}_{600}\right)$ as well as for HPLC analysis for metabolite profiling.

\subsection{Analytical methods}

Samples of culture supernatants were first filtered through $0.2 \mu \mathrm{m}$ Minisart RC membrane filters (Sartorius) and stored at $-20^{\circ} \mathrm{C}$ until analysis. The concentrations of glycerol, 1,2-PDO and ethanol in culture media were determined using a Waters HPLC system (Eschborn, Germany) equipped with two consecutive Aminex HPX-87H cation-exchange columns (Biorad, München, Germany) using 


\section{ACCEPTED MANUSCRIPT}

$5 \mathrm{mM} \mathrm{H}_{2} \mathrm{SO}_{4}$ as the mobile phase at a flow rate of $0.45 \mathrm{~mL} \mathrm{~min}^{-1}$ and a column temperature of $45^{\circ} \mathrm{C}$. Volumes of $20 \mu \mathrm{L}$ of sample were used for injection. All components were detected using the Waters 2414 Refractive Index detector. Under these conditions, the retention times were $35.6 \mathrm{~min}$ for glycerol, $45.4 \mathrm{~min}$ for 1,2-PDO and $57.7 \mathrm{~min}$ for ethanol. For quantification, standards were run in appropriate concentrations (i.e. between $1 \mathrm{~g} \mathrm{~L}^{-1}$ and $100 \mathrm{~g} \mathrm{~L}^{-1}$ for glycerol, $0.075 \mathrm{~g} \mathrm{~L}^{-1}$ and $5 \mathrm{~g} \mathrm{~L}^{-1}$ for 1,2-PDO, and $0.394 \mathrm{~g} \mathrm{~L}^{-1}$ and $39.37 \mathrm{~g} \mathrm{~L}^{-1}$ for ethanol). The obtained raw data were processed using the Breeze 2 software (Waters).

\section{RESULTS}

\subsection{Modular metabolic engineering of S. cerevisiae for the production of 1,2-PDO from glycerol}

All metabolic engineering steps in this study were conducted in the genetic background of our previously described and intensively studied haploid meiotic segregant CBS 6412-13A originating from the glycerol-utilizing wild-type S. cerevisiae strain CBS 6412 (Swinnen et al., 2016). This strain exhibiting a $\mu_{\max }$ of $0.13 \mathrm{~h}^{-1}$ in synthetic glycerol medium was the best-performing glycerol-utilizing S. cerevisiae strain available to us at the time when the current study has been initiated. As demonstrated in our previous study, the strain CBS 6412-13A solely utilizes the native FADdependent G3P pathway for glycerol catabolism (Swinnen et al., 2013).

As. S. cerevisiae does not produce 1,2-PDO naturally, the first engineering module implemented during strain construction was the expression of the so-called 'methylglyoxal pathway' (see section 1 and Figure 1). The key enzyme of this pathway is methylglyoxal synthase (MGS) and several previous studies have shown that even the sole expression of $E$. coli $m g s A$ in $S$. cerevisiae resulted in the production of small amounts of 1,2-PDO (Hoffman, 1999; Jeon et al., 2009; Lee and DaSilva, 2006). Nevertheless, the additional expression of E. coli gldA encoding a glycerol dehydrogenase was demonstrated to increase 1,2-PDO production in S. cerevisiae (Hoffman, 1999). The E. coli aldehyde reductase encoded by yqhD was also considered here to be a useful enzyme for converting methylglyoxal to 1,2-PDO. The enzyme was shown to reduce methylglyoxal in vitro (Jarboe, 2011; Lee 


\section{ACCEPTED MANUSCRIPT}

et al., 2010) and has been successfully used to improve 1,2-PDO formation in engineered C. glutamicum additionally expressing E. coli mgsA and gldA (Siebert and Wendisch, 2015). Therefore, we decided to use codon-optimized versions of the $E$. coli genes $m g s A, y q h D$ and $g / d A$ (referred to as EcmgsA, EcyqhD and EcgldA, respectively) in the current study and placed them between different S. cerevisiae promoters and terminators (see Material and Methods) in order to establish a '1,2-PDO module' in yeast. All engineered strains harboring this module (referred to as 'PDO') carried head-to-tail genomic integrations of S. cerevisiae expression cassettes for EcmgsA and EcgldA, while the expression cassette for EcyqhD was expressed from a S. cerevisiae low-copy expression vector (see section 2.5 and Table 1).

While establishing the module 'PDO', a parallel study in our laboratory revealed that the expression of a heterologous glycerol facilitator significantly improves the growth of strain CBS 6412-13A in synthetic glycerol medium (Klein et al., 2016b). As we assumed that an improved glycerol uptake might also be beneficial for 1,2-PDO production from glycerol, we decided to integrate an expression cassette bearing the coding sequence of FPS1 from Cyberlindnera jadinii (referred to here as CjFPS1) into the genome of all strains engineered for 1,2-PDO production. Accordingly, the term 'FPS' was included in the respective strain names (see section 2.5 and Table 1). The strain CBS 6412-13A expressing CjFPS1 was used as the negative reference throughout this study but CjFPS1 expression was not considered here as a separate module.

A second module addressed the co-factor supply for 1,2-PDO formation. The three step conversion from DHAP to 1,2-PDO includes two enzymatic reactions that require reducing equivalents in the form of cytosolic $\mathrm{NAD}(\mathrm{P}) \mathrm{H}$ (Figure 1). As mentioned in the introduction (section 1), the native FADdependent glycerol catabolic pathway (up to DHAP) in S. cerevisiae does not deliver cytosolic reducing equivalents. However, the pathway can be functionally replaced by an $N A D^{+}$-dependent 'DHA pathway' as recently demonstrated by Klein et al. (2016a). The respective genetic modifications included the plasmid-based heterologous expression of glycerol dehydrogenase from Ogataea parapolymorpha (Opgdh), the overexpression of endogenous dihydroxyacetone kinase (DAK1) and 


\section{ACCEPTED MANUSCRIPT}

the deletion of endogenous glycerol kinase (GUT1). These modifications are collectively referred to as module 'DHA' in the respective strain names (Table 1). This module has been supposed to strongly increase cytosolic NADH availability for 1,2-PDO production since the FAD-dependent native pathway for glycerol catabolism has been completely abolished.

An additional module addressed the need for increased availability of DHAP, the metabolic precursor for the 'methylglyoxal pathway'. The introduction of the heterologous 1,2-PDO pathway creates a metabolic branch point at which MGS must compete with the endogenous glycolytic enzyme triosephosphate isomerase (TPI) (encoded by TPI1) for their common substrate DHAP. Jung et al. (2008) showed that the deletion of the TPI1 gene in S. cerevisiae bearing a heterologous 1,2-PDO pathway resulted in increased 1,2-PDO production in a medium containing a mix of glucose, galactose and ethanol as carbon sources. However, the complete abolishment of TPI activity is not feasible when glycerol serves as the sole source of carbon since this enzyme is essential to channel the carbon flux from the substrate glycerol into the central carbon metabolism (i.e. both glycolysis and gluconeogenesis). Therefore, a strategy for downregulating TPI1 expression (without fully abolishing it) via promoter replacement was employed. In a previous study, a collection of mutant promoters with gradually decreasing strengths (based on the constitutive TEF1 promoter) had been generated (Alper et al., 2005). Nevoigt et al. (2006) used this collection to develop sets of promoter replacement cassettes allowing the exchange of any promoter in the S. cerevisiae genome with a TEF1 promoter variant of defined strength. Two weak promoters from this collection (TEFmut7 and TEFmut2) have initially been tested in the current study (Figure S2 and the respective supplementary information), and we eventually selected the weakest promoter (TEFmut2) in order to replace the native TPI1 promoter. The respective genetic modification is referred to here as module 'TPI $1_{\text {down }}$ ' (Table 1). The impact of the promoter replacement on growth of the corresponding intermediate strain in synthetic medium containing different carbon sources is shown in Figure S2 (supplementary information). These results are an indirect proof for the fact that the promoter replacement (using the TEFmut2 promoter) indeed resulted in decreased TPI activity. 


\section{ACCEPTED MANUSCRIPT}

3.2 The choice of cultivation medium strongly affected 1,2-PDO production of the engineered strains

Our initial intention was to use synthetic glycerol medium for studying the impacts of the different engineering modules on 1,2-PDO production. We decided to use urea as the source of nitrogen in our synthetic medium as it, in contrast to ammonium sulfate, does not lead to medium acidification and is therefore known to result in much higher final optical densities compared to synthetic medium with ammonium sulfate. In the urea-containing medium, the strain PDO-FPS-TPI $1_{\text {down }}$-DHA produced less than $0.2 \mathrm{~g} \mathrm{~L}^{-1}$ after $120 \mathrm{~h}$ of cultivation in synthetic glycerol medium (Figure 2A). Instead, significant concentrations of ethanol indicating an onset of fermentative metabolism were detected in the first half of the cultivation with a peak of up to $18 \mathrm{~g} \mathrm{~L}^{-1}$ after $72 \mathrm{~h}$. Later, the ethanol concentration decreased (Figure 2B).

In a previous study, Jung et al. (2011) successfully demonstrated the production of 1,2-PDO in engineered S. cerevisiae when using a complex medium (YPG containing $10 \mathrm{~g} \mathrm{~L}^{-1}$ yeast extract, $20 \mathrm{~g} \mathrm{~L}^{-1}$ peptone, $10 \mathrm{~g} \mathrm{~L}^{-1}$ glycerol and $1 \mathrm{~g} \mathrm{~L}^{-1}$ galactose). We therefore tested our advanced strain PDO-FPSTPI1 $1_{\text {down }}-\mathrm{DHA}$ in $\mathrm{YPG}_{6 \%}$ medium (10 $\mathrm{g} \mathrm{L}^{-1}$ yeast extract, $20 \mathrm{~g} \mathrm{~L}^{-1}$ peptone and $60 \mathrm{~mL} \mathrm{~L}^{-1}$ glycerol). However, only a slight improvement of 1,2-PDO production could be observed compared to synthetic medium with urea. The highest detected titer was $\sim 0.3 \mathrm{~g} \mathrm{~L}^{-1}$ after $96 \mathrm{~h}$ of cultivation. Notably, the switch to $\mathrm{YPG}_{6 \%}$ medium resulted in faster growth, glycerol consumption as well as ethanol production (Figure 2). Under these conditions, the strain accumulated up to $18 \mathrm{~g} \mathrm{~L}^{-1}$ ethanol already during the first $24 \mathrm{~h}$ of cultivation (Figure 2B).

Surprisingly, omitting peptone from our $\mathrm{YPG}_{6 \%}$ medium remarkably increased 1,2-PDO production from $\sim 0.3 \mathrm{~g} \mathrm{~L}^{-1}$ to $\sim 1.3 \mathrm{~g} \mathrm{~L}^{-1}$ where most of the product accumulated during the first $48 \mathrm{~h}$ (Figure $2 \mathrm{~A}$ ). The plausible nutrient limitation caused a much slower growth and is also reflected by a slower rate of glycerol consumption. Surprisingly, much less ethanol was produced in the absence of peptone (Figure 2B). On the basis of these results, we decided to first characterize the influence of the 


\section{ACCEPTED MANUSCRIPT}

different metabolic engineering modules on 1,2-PDO production in $\mathrm{YG}_{6 \%}$ medium and dedicated separate experiments scrutinizing the medium effects on 1,2-PDO production.

A

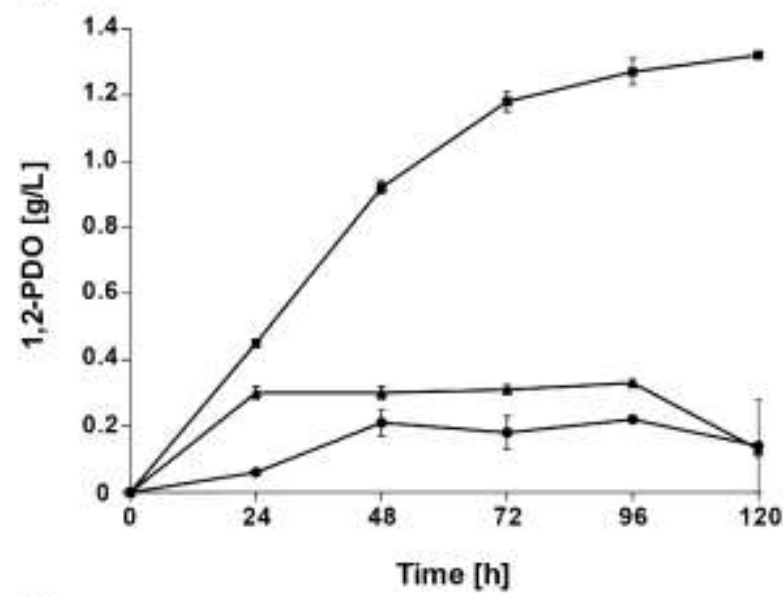

C

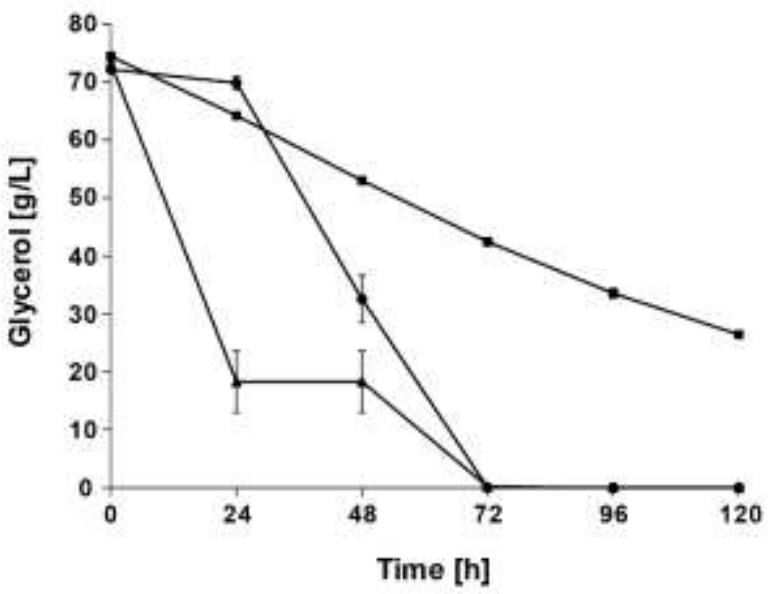

B

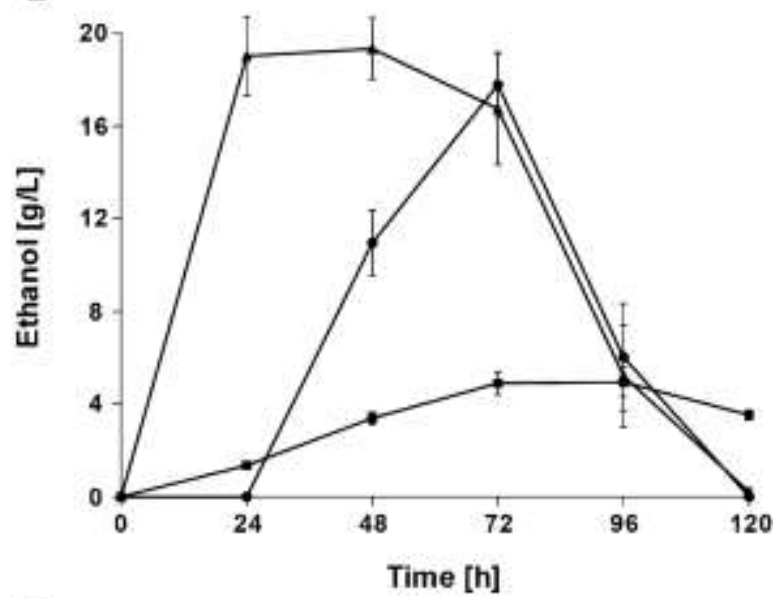

D

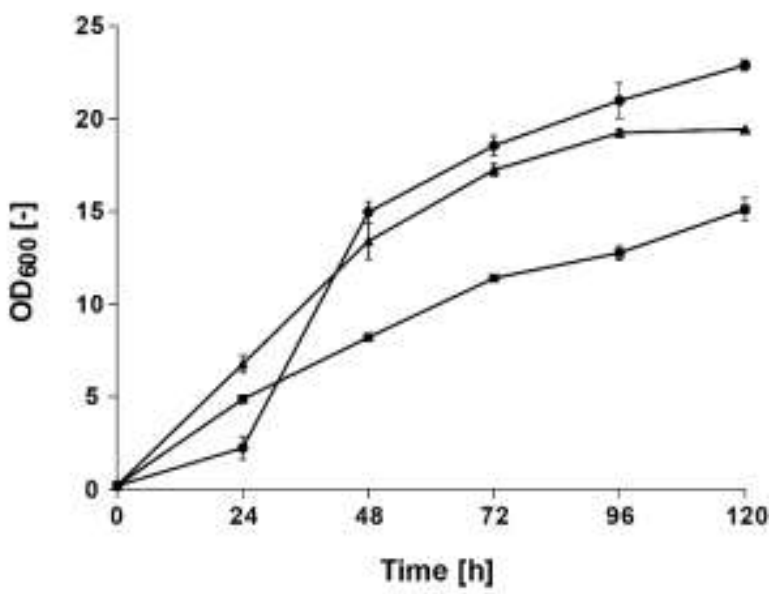
SMG

YPG YG

Figure 2 ( 2 column fitting image). Physiological characterization of strain PDO-FPS-TPI $1_{\text {down }}$-DHA in three different cultivation media containing $60 \mathrm{~mL} \mathrm{~L}^{-1}$ glycerol as the sole carbon source. The strain carried all engineering modules as described in section 3.1. Cultivations were performed in $50 \mathrm{~mL}$ shake flask cultures and culture supernatants were analyzed by HPLC for the production of 1,2-propanediol (1,2-PDO) (A), ethanol (B) and the consumption of glycerol (C). Growth was recorded by determining the optical density of the cultures at a wavelength of $600 \mathrm{~nm}\left(\mathrm{OD}_{600}\right)(\mathrm{D})$. The different media used were SMG (synthetic defined glycerol medium containing urea as the nitrogen source), YPG (complex glycerol medium containing $1 \%$ yeast extract and $2 \%$ peptone) and YG (complex glycerol medium containing $1 \%$ yeast extract). All mean values and standard deviations are derived from at least three biological replicates. 


\section{ACCEPTED MANUSCRIPT}

3.3 The combination of modules DHA and TPI $1_{\text {down }}$ was crucial to achieve the highest concentration of 1,2-PDO in $\mathrm{YG}_{6 \%}$ medium

As mentioned in section $3.2, \mathrm{YG}_{6 \%}$ medium was used in order to characterize all constructed strains and dissect the impacts of the individual modules and their combinations on 1,2-PDO production and the cells' physiology (Figure 3).

A

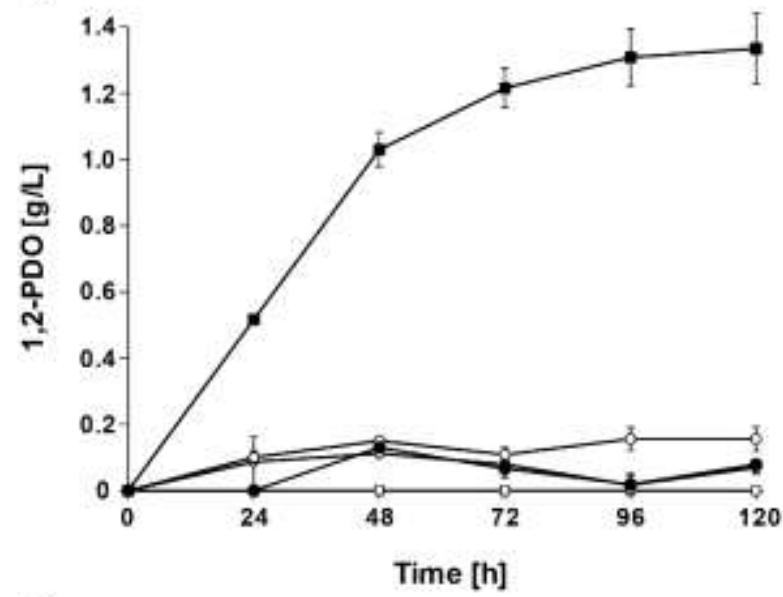

C

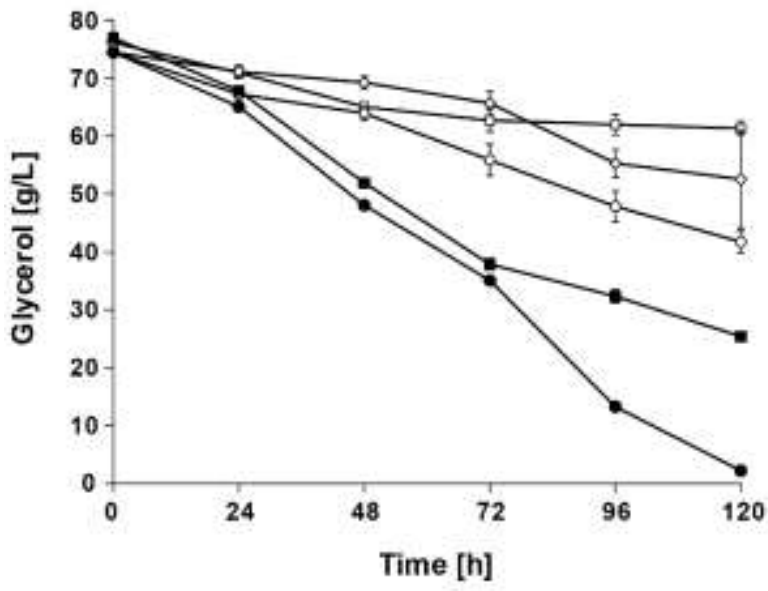

B

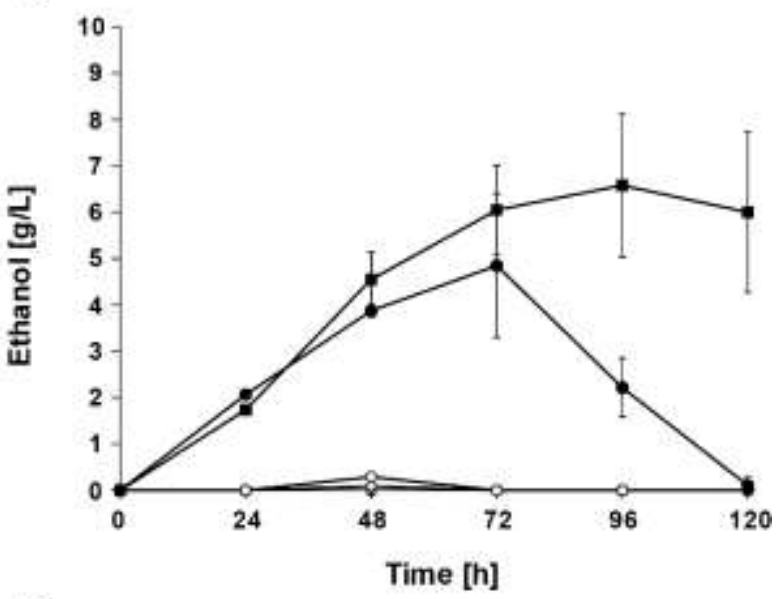

D

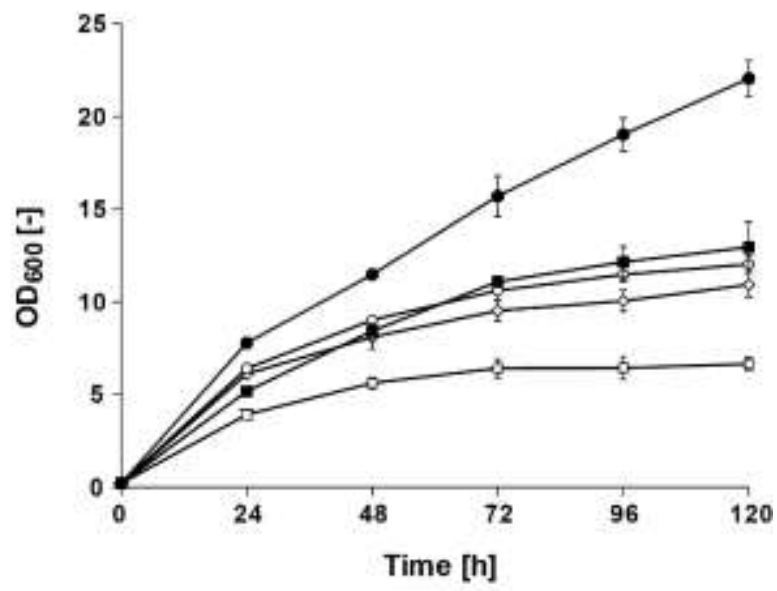

\section{FPS \\ PDO-FPS -O-PDO-FPS-TPI1 down \\ PDO-FPS - DHA \\ PDO - FPS - TPI1down - DHA}

Figure 3 ( 2 column fitting image). Physiological characterization of $S$. cerevisiae strains (derivatives of CBS 6412-13A) engineered for 1,2-PDO production from glycerol in YG medium containing $60 \mathrm{~mL} \mathrm{~L}^{-1}$ glycerol. Besides an expression cassette for the glycerol facilitator encoded by CjFPS1 ('FPS'), the analyzed strains carried the engineering module 'PDO' alone or in combination with the modules 'DHA' and 'TPI $1_{\text {down' }}$ as described in section 3.1. Cultivations were performed in $50 \mathrm{~mL}$ shake flask cultures and culture supernatants were analyzed by HPLC for the production of 1,2-PDO (A), ethanol (B) and the consumption of glycerol (C). 


\section{ACCEPTED MANUSCRIPT}

Growth was recorded by optical density measurements at $600 \mathrm{~nm}$ (D). Mean values and standard deviations were determined from at least three biological replicates.

Compared to the control strain FPS, all strains bearing the '1,2-PDO module' produced at least small amounts of 1,2-PDO (Figure $3 \mathrm{~A}$ ). Interestingly, neither the sole downregulation of TPI1 expression (module 'TPI $1_{\text {down }}$ ') nor the sole glycerol catabolic pathway replacement (module 'DHA') in the genetic background of the strain PDO-FPS resulted in a significant improvement of the 1,2-PDO titer. Only the combination of these two metabolic engineering modules with the '1,2-PDO module' considerably improved 1,2-PDO production from less than $0.2 \mathrm{~g} \mathrm{~L}^{-1}$ to $\sim 1.3 \mathrm{~g} \mathrm{~L}^{-1}$ when measured after $120 \mathrm{~h}$ of cultivation. The results demonstrate that both above-mentioned modules were essential for 1,2-PDO production from glycerol.

The glycerol catabolic pathway replacement in strain PDO-FPS improved the strain's glycerol consumption (Figure 3C, strains PDO-FPS-DHA and PDO-FPS-TPI $1_{\text {down }}-\mathrm{DHA}$ ) indicating that the DHA pathway allows a better flux from glycerol to DHAP compared to the G3P pathway present in the wild-type strain. In strain PDO-FPS-DHA, the increased glycerol consumption coincides with a faster growth, while the presence of the module 'TPI $1_{\text {down }}$ ' seems to abrogate the growth supporting effect of module 'DHA' as visible in the growth curve for strain PDO-FPS-TPI $1_{\text {down }}-$ DHA (Figure 3D).

Our data clearly show that the genetic manipulation mainly responsible for the production of ethanol observed in $\mathrm{YG}_{6 \%}$ medium is the replacement of the endogenous glycerol catabolic pathway by the NADH-generating DHA pathway (Figure 3B). A switch to fermentative metabolism (visible by ethanol formation) is plausible when considering the excess of cytosolic NADH generated by the latter pathway. Again, the ethanol production was particularly high in the first half of the cultivation period, while its concentration declined during the second part of the cultivation (Figure 3B). During the second phase of cultivation, glycerol was still present and its concentration continued to decrease. Interestingly, the superior growth of strain PDO-FPS-DHA coincided with a strong decrease in ethanol concentration while the respective reduction in ethanol concentration during the second 


\section{ACCEPTED MANUSCRIPT}

cultivation half was very low in the strain with additional downregulation of TPI1 expression (Figure 3B).

\subsection{Implementation of a second expression cassette for $E$. coli MGS and analysis in controlled batch} cultivation in bioreactors

In the previous section, it was shown that the modules for cofactor-supply and carbon flux redirection ('DHA' and 'TPI $1_{\text {down }}$ ', respectively) were essential genetic manipulations to increase the capacity of S. cerevisiae to produce 1,2-PDO from glycerol. The next question that arose was whether the flux into the 1,2-PDO pathway was rate-controlling for 1,2-PDO production in the strain PDO-FPSTPI $1_{\text {down }}-$ DHA. At this stage, we decided to construct a strain that carries all genetic modifications within its genome (including the implementation of an additional expression cassette for $E$. coli $m g s A)$ resulting in strain PDO-FPS-TPI $1_{\text {down }}$-DHA $E c m g s A_{2}$. This also facilitated the physiological characterization in larger volumes since no antibiotic had to be added anymore. When this strain was analyzed in shake flasks under the same conditions as used before, the additional EcmgsA expression cassette resulted in increased 1,2-PDO production ( $2.5 \mathrm{~g} \mathrm{~L}^{-1}$ after $140 \mathrm{~h}$ of cultivation) while the maximum ethanol titer decreased from $\sim 6.6 \mathrm{~g} \mathrm{~L}^{-1}$ to $\sim 3.8 \mathrm{~g} \mathrm{~L}^{-1}$ as shown in Figure $\mathrm{S} 3$ (supplementary information).

As a next step, the strain PDO-FPS-TPI1 down $-\mathrm{DHA} E \mathrm{E} m g s A_{2}$ was characterized under controlled conditions in batch cultivations in bioreactors using the same medium $\left(\mathrm{YG}_{6 \%}\right)$. In general, the strain's physiology was very similar to that observed in shake flask cultures. However, the controlled cultivation conditions allowed the cells to reach higher optical densities than in shake flasks. The maximum 1,2-PDO titer reached was $\sim 4.3 \mathrm{~g} \mathrm{~L}^{-1}$ (Figure 4). This has been the highest reported 1,2-PDO titer obtained by fermentations with engineered S. cerevisiae. 


\section{ACCEPTED MANUSCRIPT}

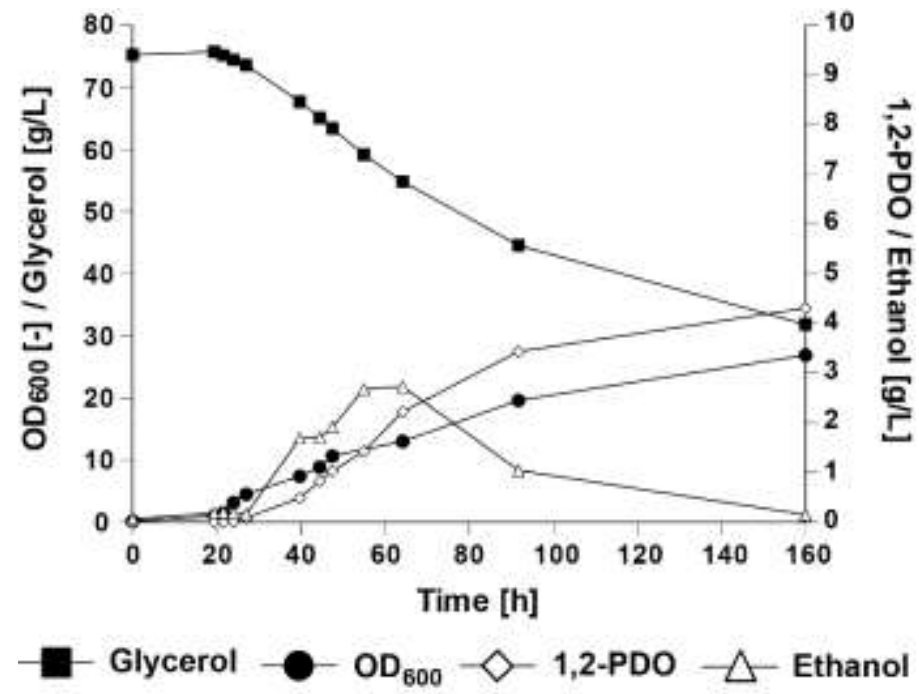

Figure 4 (single column fitting image). Physiological characterization of strain PDO-FPS-TPI1 $1_{\text {down }}-$ DHA$E c m g s A_{2}$ in batch cultivations in $1 \mathrm{~L}$ bioreactors using YG medium containing $60 \mathrm{~mL} \mathrm{~L}^{-1}$ glycerol as the carbon source. The analyzed strain carried genomic integrations of all engineering modules, i.e. besides the glycerol facilitator encoded by CjFPS1 ('FPS'), the methylglyoxal pathway ('PDO') including a second expression cassette for $E$. coli $m g s A\left(E c m g s A_{2}\right)$, the glycerol catabolic pathway replacement ('DHA') and the replacement of the native TPI1 promoter by the weak TEF1 promoter variant TEFmut2 ('TPI $1_{\text {down }}$ '). One representative out of three experiments is shown.

\subsection{1,2-PDO production in buffered synthetic medium with ammonium sulfate as a carbon source}

The rather surprising results concerning the influence of the medium composition on the observable product pattern encouraged us to conduct a number of experiments in order to gain further insight into this phenomenon. The results (data not shown) revealed that the impact of medium components is very complex and most probably even strain dependent. Further experiments have to be carried out in order to dissect and understand the observed medium effects. Among the already performed experiments regarding medium effects, there has been one that was set to test whether glycerol fermentation is also visible in synthetic glycerol medium with ammonium sulfate as the nitrogen source as soon as a buffer is added and thereby rapid medium acidification is avoided resulting in significantly higher optical densities. This question came up after we detected significant ethanol production in our strains with the 'DHA' module (sections 3.2 and 3.3), a fact that has not 


\section{ACCEPTED MANUSCRIPT}

been recognized in our previous study regarding DHA pathway strains (Klein et al., 2016a). However, the earlier study was performed using unbuffered synthetic medium with ammonium sulfate as the nitrogen source. Therefore, $100 \mathrm{mM}$ potassium phthalate buffer was added to synthetic medium containing either ammonium sulfate or urea. This allowed a direct comparison of the two nitrogen sources. The respective experiment was performed with the above-mentioned strain PDO-FPSTPI $1_{\text {down }}$-DHA EcmgsA $A_{2}$ carrying two expression cassettes for EcmgsA and in which all genetic modifications were stably integrated into the genome. To our huge surprise, the buffered ammonium sulfate containing medium led to a significant formation of 1,2-PDO and a comparably low ethanol production which was in clear contrast to the results obtained for the urea-containing medium (Figure 5). The remarkable difference with regard to the 1,2-PDO/ethanol ratio between the two nitrogen sources (Figure 5A and B) cannot simply be explained by the obtained culture density (Figure 5A). In fact, the used nitrogen source seemed to have a (so far unknown) impact on the carbon flux distribution in our engineered strains. 
A

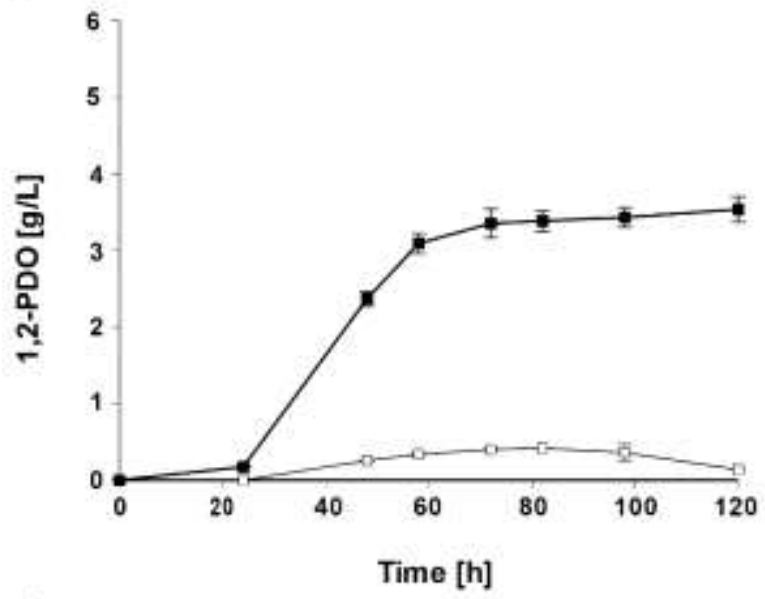

C

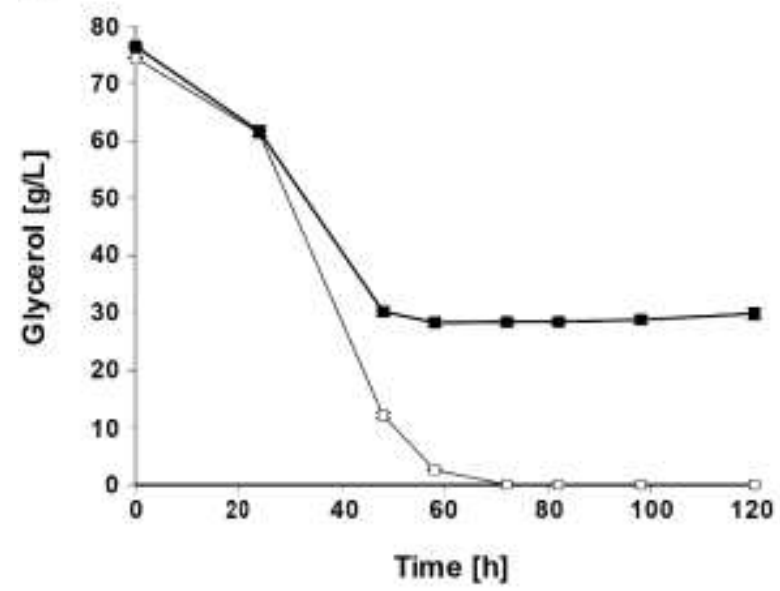

Urea
B

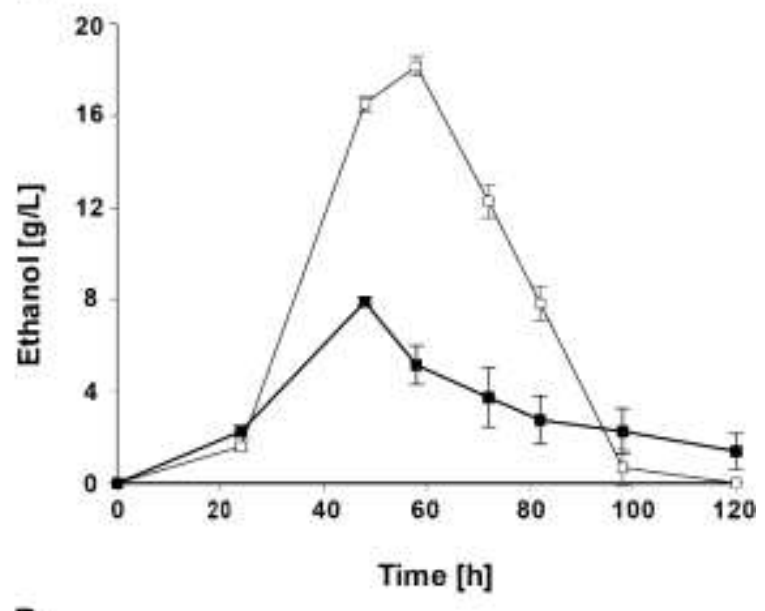

D

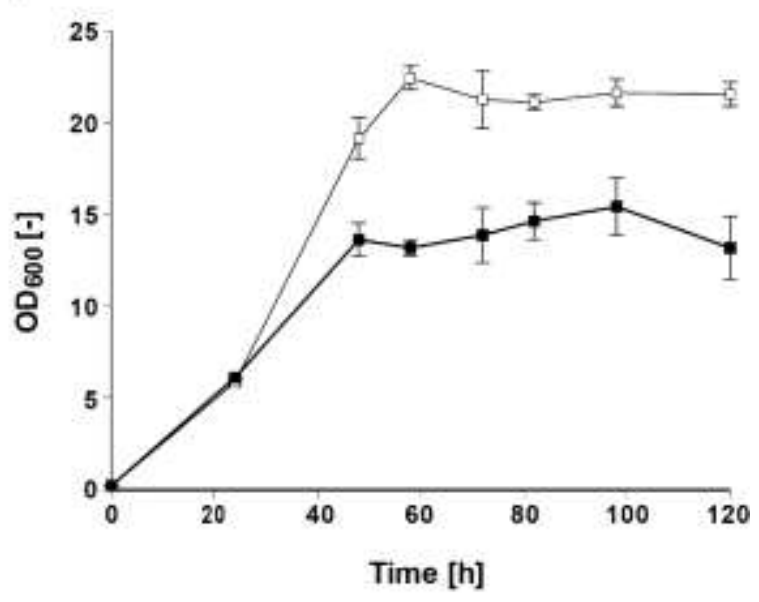

Ammonium sulfate

Figure 5 ( 2 column fitting image). Influence of the used nitrogen source on the physiology of the S. cerevisiae strain PDO-FPS-TPI $1_{\text {down }}$-DHA-EcmgsA $A_{2}$ engineered for 1,2-PDO production from glycerol in synthetic medium buffered with $100 \mathrm{mM}$ potassium phthalate buffer. The initial pH of the media was set to 5.0. Cultivations were performed in $50 \mathrm{~mL}$ shake flask cultures and culture supernatants were analyzed by HPLC for the production of 1,2-PDO (A), ethanol (B) and the consumption of glycerol (C). Growth was recorded by optical density measurements at $600 \mathrm{~nm}$ (D). Mean values and standard deviations were determined from at least three biological replicates. 


\section{ACCEPTED MANUSCRIPT}

\section{Discussion}

In this study, a novel metabolic engineering approach was used to produce 1,2-PDO in S. cerevisiae from glycerol as the sole carbon source. A previous attempt to establish 1,2-PDO production from glycerol in S. cerevisiae (equipped with the 'methylglyoxal pathway') focused on the overexpression of GUT1 and GUT2 which encode the enzymes for the native (FAD-dependent) glycerol catabolic pathway (Jung et al., 2011). However, the electrons stemming from glycerol oxidation through this pathway are eventually transferred to oxygen via the respiratory chain. In contrast, our current approach was based on the complete replacement of the endogenous G3P pathway by the NAD+dependent DHA pathway according to Klein et al. (2016a). This replacement has been supposed to provide cytosolic reducing equivalents from glycerol and facilitate the formation of fermentation products such as 1,2-PDO. The current study demonstrates that the module ' $\mathrm{DHA}^{\prime}$ ' indeed increased the formation of the target product at least when TPI expression was reduced in parallel thereby increasing the availability of the precursor DHAP. Notably, downregulation of TPI expression alone did not result in significant 1,2-PDO formation. These results underline the importance of providing sufficient concentrations of both pre-cursor and co-factor for the successful re-direction of metabolic fluxes. The fact that 1,2-PDO production in the presence of increased pre-cursor and co-factor could be even further increased by a second $m g s A$ expression cassette demonstrates that the flux through methylglyoxal synthase has been the rate-controlling step in the respective genetic background and might be one important target for further strain improvement.

As already mentioned, the 1,2-PDO titer of $>4 \mathrm{~g} / \mathrm{l}$ achieved in the current study has been the highest reported for S.cerevisiae. This titer obtained in the bioreactor experiment in YG medium corresponded to a maximum product yield of $\sim 129 \pm 21 \mathrm{mg}$ per g glycerol consumed. Jung et al. (2011) reported a 1,2-PDO yield of $213 \mathrm{mg}$ per g glycerol consumed. However, their experiments were performed in modified YEPD medium (yeast extract $1 \%$, peptone $2 \%$, glycerol $1 \%$ and galactose $0.1 \%)$. Although the galactose was only added for induction of gene expression in the latter study, its utilization as a carbon source may have falsified the calculated yields. Moreover, ingredients of the 


\section{ACCEPTED MANUSCRIPT}

added peptone might have also been used as minor carbon sources. These facts hamper a reliable comparison with our results.

Regarding the pathway from DHAP to 1,2-PDO, there are several possible metabolic routes which may result in either R- or S-1,2-PDO (Figure 1). However, we have not dissected in our analytics so far whether only one or both enantiomers were formed by our engineered S. cerevisiae strains. In addition, it has been difficult to draw a strong hypothesis on the actual carbon flux solely based on published knowledge. First, the genes EcgldA and EcyqhD encode for enzymes with relatively broad substrate specificities (Jarboe, 2011; St. Martin et al., 1977; Tang et al., 1979). Second, the gene Opgdh originally used for the module 'DHA' might also contribute to the reductive reactions from methylglyoxal to 1,2-PDO to a certain degree since its expression (instead of EcgldA) in a $\mathrm{PDO}_{\min }$ strain also increased 1,2-PDO formation as compared to the sole expression of EcmgsA (results not shown). Third, endogenous (probably rather unspecific) reductases of the yeast $S$. cerevisiae have also to be considered to be active in the respective chemical conversions as mentioned in section 1 . Nevertheless, our 1,2-PDO producing strains formed a significant amount of an additional product as visible by a conspicuous peak in the HPLC (data not shown). HPLC analysis of the retention times of the pathway intermediates methylglyoxal, R-/S-lactaldehyde and acetol indicated that acetol might be the compound that was accumulated by our 1,2-PDO producer. The retention time of lactaldehyde under the employed conditions is close to that of glycerol and the latter was still present in relatively large concentrations in the medium at the end of the cultivation period in most of our experiments. Therefore, a peak for lactaldehyde is assumed to partially overlap with the peak for glycerol. At least, we did not recognize any shoulder for the glycerol peak in our chromatograms. Notably, other authors expressing the same $E$. coli genes for 1,2-PDO formation in C. glutamicum also reported significant acetol formation (Siebert and Wendisch, 2015), while Altaras and Cameron (1999) found R-lactaldehyde as an intermediate in an E. coli strain overexpressing E. coli $m g s A$ and gldA. Future work including metabolomics will focus on a more comprehensive characterization of the carbon flow in our engineered strains. 


\section{ACCEPTED MANUSCRIPT}

Glycerol has been considered as a carbon source that is solely metabolized in a respiratory manner in

S. cerevisiae. The full dependence of glycerol catabolism on respiration in wild-type S. cerevisiae strains can be explained by the fact that the electrons are directly transferred by Gut2 via $\mathrm{FADH}_{2}$ to the mitochondrial respiratory chain. This also holds for strains which display higher growth rates (and glycolytic flux) in synthetic glycerol medium than the majority of wild-type $S$. cerevisiae strains. In fact, Ochoa-Estopier et al. (2011) comprehensively studied an evolved derivative of CBS 8066 (FL20) that exhibited a $\mu_{\max }$ of $0.20 \mathrm{~h}^{-1}$ in synthetic glycerol medium in a controlled bioreactor setup and no reduced fermentation products such as ethanol were detected. Similarly, no fermentation products were detected in shake-flask cultivations using our non-engineered wild-type S. cerevisiae strain CBS 6412-13A which is able to grow in synthetic glycerol medium with a $\mu_{\max }$ of $0.13 \mathrm{~h}^{-1}$ via the G3P pathway (data not shown).

In the view of the current study it is, however, important to note that the applied glycerol catabolic pathway replacement strategy rendered the strain's glycerol catabolism solely dependent on NAD'. This has been assumed to result in surplus cytosolic NADH and to facilitate the production of fermentation products in our strains such as 1,2-PDO (heterologous pathway) and/or ethanol (S. cerevisiae's major native fermentation product). Indeed, our data indicates that cells, which depend on the DHA pathway for glycerol catabolism, at least partially ferment the glycerol, even though both the degree of fermentation and the ratio between 1,2-PDO and ethanol formation are strain- and medium dependent.

So far, we cannot dissect whether the surplus of cytosolic NADH caused the fermentative metabolism of glycerol right at the beginning of the cultivation or whether any kind of nutrient or oxygen limitation was an additional requirement for the metabolic switch. At least, one has to note that ethanol formation was also visible in the bioreactor experiment employing the $\mathrm{YG}_{6 \%}$ medium where air was flushed with a rate of $1 \mathrm{vvm}$ (stirring rate $800 \mathrm{rpm}$ ) into the culture. The underlying basis why 1,2-PDO is produced in significant amounts in $\mathrm{YG}_{6 \%}$ medium (and in the buffered ammonium sulfate-containing synthetic medium) while only trace amounts of the target product 


\section{ACCEPTED MANUSCRIPT}

were formed in synthetic medium with urea as a nitrogen source and in $\mathrm{YPG}_{6 \%}$ medium (where ethanol is the prominent fermentation product) has not been clarified yet. By unknown reasons, the engineered 1,2-PDO pathway can probably better compete with the endogenous ethanol formation pathway in the first two media.

It has been very intriguing that the concentration of ethanol in the medium started to decrease at a certain point in time. In general, the actual ethanol concentration could have been affected by three different processes which might, at least partly, have occurred in parallel: i) ethanol formation, ii) evaporation and iii) ethanol consumption. Evaporation was tested in a parallel experiment using medium without cells and an initial ethanol concentration of $25 \mathrm{~g} \mathrm{~L}^{-1}$. Under the applied conditions, the ethanol evaporated with a maximum rate of $\sim 0.10 \mathrm{~g} \mathrm{~L}^{-1} \mathrm{~h}^{-1}$. This rate was lower than observed for the decrease in ethanol concentration for the analyzed cultures which varied between 0.16 and 0.44 $\mathrm{g} \mathrm{L}^{-1} \mathrm{~h}^{-1}$. Although its impact can definitely not be neglected, evaporation can therefore not completely explain the observed decrease in ethanol concentration in our experiments. Thus, it can be concluded that ethanol might have been consumed together with glycerol and even further when glycerol was completely depleted (Figure 2). Assuming the actual occurrence of ethanol consumption by the cells, the observed decrease of the ethanol titer in the second cultivation period may have been caused by the fact that ethanol production by fermentation stopped (or at least slowed down) while respiratory ethanol utilization continued or even increased. A reduction in glycerol consumption rate at a later stage in the cultivation was also visible. Although we do not have a plausible explanation for this result, it might underpin our hypothesis with regard to a certain overflow metabolism at increased glycerol consumption rates. The lower glycerol consumption rate might have resulted in less NADH generation and the rate of respiration might have been sufficient to reoxidize the formed NADH explaining why the cells did not need fermentation at the later phase of cultivation. Nevertheless, we cannot exclude that regulatory mechanisms changed the rate of fermentation and/or respiration at this point in time and further work will be necessary to clarify this aspect. 


\section{ACCEPTED MANUSCRIPT}

The data presented in the current manuscript demonstrate that $S$. cerevisiae can be engineered to produce significant concentrations of 1,2-PDO ( $>4 \mathrm{~g} \mathrm{~L}^{-1}$ in a controlled bioreactor set-up). Our study shows that both metabolic precursor and co-factor supply are essential for target product formation. The work also demonstrates the crucial impact of the medium composition and the experimental conditions on the metabolic mode and, thus product titer and yield. We therefore believe that there is still a lot of room for further improving 1,2-PDO production in S. cerevisiae.

\section{Acknowledgements}

This work was funded through the ERA-NET scheme of the $7^{\text {th }}$ EU Framework Program (IPCRES; German Federal Ministry of Education and Research, Project No. 031A344). We thank Johan M. Thevelein (KU Leuven, Belgium) for kindly providing us with the plasmid pGG119 and Solvejg Sevecke, Martina Carrillo, and Joeline Xiberras for technical support. 


\section{REFERENCES}

Akada, R., Hirosawa, I., Kawahata, M., Hoshida, H., Nishizawa, Y., 2002. Sets of integrating plasmids and gene disruption cassettes containing improved counter-selection markers designed for repeated use in budding yeast. Yeast. 19, 393-402.

Alper, H., Fischer, C., Nevoigt, E., Stephanopoulos, G., 2005. Tuning genetic control through promoter engineering. Proc Natl Acad Sci U S A. 102, 12678-83.

Altaras, N. E., Cameron, D. C., 1999. Metabolic engineering of a 1,2-propanediol pathway in Escherichia coli. Appl Environ Microbiol. 65, 1180-5.

Altaras, N. E., Cameron, D. C., 2000. Enhanced production of (R)-1,2-propanediol by metabolically engineered Escherichia coli. Biotechnol Prog. 16, 940-6.

Badia, J., Ros, J., Aguilar, J., 1985. Fermentation mechanism of fucose and rhamnose in Salmonella typhimurium and Klebsiella pneumoniae. J Bacteriol. 161, 435-7.

Barbier, G. G., Ladd, J. L., Campbell, E. R., 2011. Genetic Modification of Pichia pastoris for Production of Propylene Glycol from Glycerol. International Journal of Genetic Engineering. 1, 6-13.

Bennett, G. N., San, K. Y., 2001. Microbial formation, biotechnological production and applications of 1,2-propanediol. Appl Microbiol Biotechnol. 55, 1-9.

Berrios-Rivera, S. J., San, K. Y., Bennett, G. N., 2003. The effect of carbon sources and lactate dehydrogenase deletion on 1,2-propanediol production in Escherichia coli. J Ind Microbiol Biotechnol. 30, 34-40.

Borodina, I., Nielsen, J., 2014. Advances in metabolic engineering of yeast Saccharomyces cerevisiae for production of chemicals. Biotechnol J. 9, 609-20.

Boronat, A., Aguilar, J., 1979. Rhamnose-induced propanediol oxidoreductase in Escherichia coli: purification, properties, and comparison with the fucose-induced enzyme. J Bacteriol. 140, 320-6.

Boronat, A., Aguilar, J., 1981. Metabolism of L-fucose and L-rhamnose in Escherichia coli: differences in induction of propanediol oxidoreductase. J Bacteriol. 147, 181-5.

Burk, M. J., Van Dien, S., 2016. Biotechnology for Chemical Production: Challenges and Opportunities. Trends Biotechnol. 34, 187-90.

Cameron, D. C., Altaras, N. E., Hoffman, M. L., Shaw, A. J., 1998. Metabolic engineering of propanediol pathways. Biotechnol Prog. 14, 116-25.

Cameron, D. C., Cooney, C. L., 1986. A Novel Fermentation - the Production of R(-)-1,2-Propanediol and Acetol by Clostridium-Thermosaccharolyticum. Bio-Technology. 4, 651-654.

Chao, R., Yuan, Y., Zhao, H., 2015. Recent advances in DNA assembly technologies. FEMS Yeast Res. $15,1-9$.

Clomburg, J. M., Gonzalez, R., 2011. Metabolic engineering of Escherichia coli for the production of 1,2-propanediol from glycerol. Biotechnol Bioeng. 108, 867-79.

da Silva, G. P., Mack, M., Contiero, J., 2009. Glycerol: a promising and abundant carbon source for industrial microbiology. Biotechnol Adv. 27, 30-9.

David, F., Siewers, V., 2015. Advances in yeast genome engineering. FEMS Yeast Res. 15, 1-14.

Dowd, M. K., Johansen, S. L., Cantarella, L., Reilly, P. J., 1994. Low-Molecular-Weight Organic Composition of Ethanol Stillage from Sugarcane Molasses, Citrus Waste, and Sweet Whey. Journal of Agricultural and Food Chemistry. 42, 283-288.

Flagfeldt, D. B., Siewers, V., Huang, L., Nielsen, J., 2009. Characterization of chromosomal integration sites for heterologous gene expression in Saccharomyces cerevisiae. Yeast. 26, 545-51.

Gibson, D. G., Young, L., Chuang, R. Y., Venter, J. C., Hutchison, C. A., 3rd, Smith, H. O., 2009. Enzymatic assembly of DNA molecules up to several hundred kilobases. Nat Methods. 6, 3435.

Gietz, R. D., Schiestl, R. H., Willems, A. R., Woods, R. A., 1995. Studies on the transformation of intact yeast cells by the LiAc/SS-DNA/PEG procedure. Yeast. 11, 355-60. 


\section{ACCEPTED MANUSCRIPT}

Gonzalez, R., Murarka, A., Dharmadi, Y., Yazdani, S. S., 2008. A new model for the anaerobic fermentation of glycerol in enteric bacteria: trunk and auxiliary pathways in Escherichia coli. Metab Eng. 10, 234-45.

Gueldener, U., Heck, S., Fielder, T., Beinhauer, J., Hegemann, J. H., 1996. A new efficient gene disruption cassette for repeated use in budding yeast. Nucleic Acids Res. 24, 2519-24.

Gueldener, U., Heinisch, J., Koehler, G. J., Voss, D., Hegemann, J. H., 2002. A second set of loxP marker cassettes for Cre-mediated multiple gene knockouts in budding yeast. Nucleic Acids Res. 30, e23.

Hoffman, C. S., Winston, F., 1987. A ten-minute DNA preparation from yeast efficiently releases autonomous plasmids for transformation of Escherichia coli. Gene. 57, 267-72.

Hoffman, M. L., Metabolic engineering of 1,2-Propanediol production in Saccharomyces cerevisiae. University of Wisconsin--Madison, 1999, pp. xi, 218 leaves.

Hong, K. K., Nielsen, J., 2012. Metabolic engineering of Saccharomyces cerevisiae: a key cell factory platform for future biorefineries. Cell Mol Life Sci. 69, 2671-90.

Huang, K., Rudolph, F. B., Bennett, G. N., 1999. Characterization of methylglyoxal synthase from Clostridium acetobutylicum ATCC 824 and its use in the formation of 1, 2-propanediol. Appl Environ Microbiol. 65, 3244-7.

Jain, R., Huang, J., Yuan, Q., Yan, Y., 2015a. Engineering microaerobic metabolism of E. coli for 1,2propanediol production. J Ind Microbiol Biotechnol. 42, 1049-55.

Jain, R., Sun, X., Yuan, Q., Yan, Y., 2015b. Systematically engineering Escherichia coli for enhanced production of 1,2-propanediol and 1-propanol. ACS Synth Biol. 4, 746-56.

Jarboe, L. R., 2011. YqhD: a broad-substrate range aldehyde reductase with various applications in production of biorenewable fuels and chemicals. Appl Microbiol Biotechnol. 89, 249-57.

Jeon, E., Lee, S., Kim, D., Yoon, H., Oh, M., Park, C., Lee, J., 2009. Development of a Saccharomyces cerevisiae strain for the production of 1,2-propanediol by gene manipulation. Enzyme and Microbial Technology. 45, 42-47.

Johnson, D. T., Taconi, K. A., 2007. The glycerin glut: Options for the value-added conversion of crude glycerol resulting from biodiesel production. Environmental Progress. 26, 338-348.

Jung, J. Y., Choi, E. S., Oh, M. K., 2008. Enhanced production of 1,2-propanediol by tpi1 deletion in Saccharomyces cerevisiae. J Microbiol Biotechnol. 18, 1797-802.

Jung, J. Y., Yun, H. S., Lee, J., Oh, M. K., 2011. Production of 1,2-propanediol from glycerol in Saccharomyces cerevisiae. J Microbiol Biotechnol. 21, 846-53.

Karinen, R. S., Krause, A. O. I., 2006. New biocomponents from glycerol. Applied Catalysis A: General. 306, 128-133.

Klein, M., Carrillo, M., Xiberras, J., Islam, Z. U., Swinnen, S., Nevoigt, E., 2016a. Towards the exploitation of glycerol's high reducing power in Saccharomyces cerevisiae-based bioprocesses. Metab Eng.

Klein, M., Islam, Z.-u., Knudsen, P. B., Carrillo, M., Swinnen, S., Workman, M., Nevoigt, E., 2016b. The expression of glycerol facilitators from various yeast species improves growth on glycerol of Saccharomyces cerevisiae. Metabolic Engineering Communications. 3, 252-257.

Klein, M., Swinnen, S., Thevelein, J., Nevoigt, E., 2016c. Glycerol metabolism and transport in yeast and fungi: Established knowledge and ambiguities. Environ Microbiol.

Lee, C., Kim, I., Lee, J., Lee, K. L., Min, B., Park, C., 2010. Transcriptional activation of the aldehyde reductase YqhD by YqhC and its implication in glyoxal metabolism of Escherichia coli K-12. J Bacteriol. 192, 4205-14.

Lee, M. J., Brown, I. R., Juodeikis, R., Frank, S., Warren, M. J., 2016. Employing bacterial microcompartment technology to engineer a shell-free enzyme-aggregate for enhanced 1,2propanediol production in Escherichia coli. Metab Eng. 36, 48-56.

Lee, W., DaSilva, N. A., 2006. Application of sequential integration for metabolic engineering of 1,2propanediol production in yeast. Metab Eng. 8, 58-65.

Li, H., Liao, J. C., 2013. Engineering a cyanobacterium as the catalyst for the photosynthetic conversion of CO2 to 1,2-propanediol. Microb Cell Fact. 12, 4. 


\section{ACCEPTED MANUSCRIPT}

Mattam, A. J., Clomburg, J. M., Gonzalez, R., Yazdani, S. S., 2013. Fermentation of glycerol and production of valuable chemical and biofuel molecules. Biotechnol Lett. 35, 831-42.

Merico, A., Ragni, E., Galafassi, S., Popolo, L., Compagno, C., 2011. Generation of an evolved Saccharomyces cerevisiae strain with a high freeze tolerance and an improved ability to grow on glycerol. J Ind Microbiol Biotechnol. 38, 1037-44.

Murarka, A., Dharmadi, Y., Yazdani, S. S., Gonzalez, R., 2008. Fermentative utilization of glycerol by Escherichia coli and its implications for the production of fuels and chemicals. Appl Environ Microbiol. 74, 1124-35.

Naito, Y., Hino, K., Bono, H., Ui-Tei, K., 2015. CRISPRdirect: software for designing CRISPR/Cas guide RNA with reduced off-target sites. Bioinformatics. 31, 1120-3.

Nevoigt, E., 2008. Progress in metabolic engineering of Saccharomyces cerevisiae. Microbiol Mol Biol Rev. 72, 379-412.

Nevoigt, E., Genetically modified yeast with improved glycerol catabolism. Vol. DE112015003261 (A5), Germany, 2017.

Nevoigt, E., Kohnke, J., Fischer, C. R., Alper, H., Stahl, U., Stephanopoulos, G., 2006. Engineering of promoter replacement cassettes for fine-tuning of gene expression in Saccharomyces cerevisiae. Appl Environ Microbiol. 72, 5266-73.

Niimi, S., Suzuki, N., Inui, M., Yukawa, H., 2011. Metabolic engineering of 1,2-propanediol pathways in Corynebacterium glutamicum. Appl Microbiol Biotechnol. 90, 1721-9.

Ochoa-Estopier, A., Lesage, J., Gorret, N., Guillouet, S. E., 2011. Kinetic analysis of a Saccharomyces cerevisiae strain adapted for improved growth on glycerol: Implications for the development of yeast bioprocesses on glycerol. Bioresour Technol. 102, 1521-7.

Shelley, S., 2007. A renewable route to propylene glycol. Chem Eng Prog. 103, 6-9.

Siebert, D., Wendisch, V. F., 2015. Metabolic pathway engineering for production of 1,2-propanediol and 1-propanol by Corynebacterium glutamicum. Biotechnol Biofuels. 8, 91.

St. Martin, E. J., Freedberg, W. B., Lin, E. C., 1977. Kinase replacement by a dehydrogenase for Escherichia coli glycerol utilization. J Bacteriol. 131, 1026-8.

Steensma, H. Y., Ter Linde, J. J., 2001. Plasmids with the Cre-recombinase and the dominant nat marker, suitable for use in prototrophic strains of Saccharomyces cerevisiae and Kluyveromyces lactis. Yeast. 18, 469-72.

Suzuki, T., Onishi, H., 1968. Aerobic Dissimilation of L-Rhamnose and Production of L-Rhamnonic Acid and 1,2-Propanediol by Yeasts. Agricultural and Biological Chemistry. 32, 888-\&.

Swinnen, S., Ho, P. W., Klein, M., Nevoigt, E., 2016. Genetic determinants for enhanced glycerol growth of Saccharomyces cerevisiae. Metab Eng. 36, 68-79.

Swinnen, S., Klein, M., Carrillo, M., McInnes, J., Nguyen, H. T., Nevoigt, E., 2013. Re-evaluation of glycerol utilization in Saccharomyces cerevisiae: characterization of an isolate that grows on glycerol without supporting supplements. Biotechnol Biofuels. 6, 157.

Tang, C. T., Ruch, F. E., Jr., Lin, C. C., 1979. Purification and properties of a nicotinamide adenine dinucleotide-linked dehydrogenase that serves an Escherichia coli mutant for glycerol catabolism. J Bacteriol. 140, 182-7.

Thompson, J. C., He, B. B., 2006. Characterization of crude glycerol from biodiesel production from multiple feedstocks. Applied Engineering in Agriculture. 22, 261-265.

Tran-Din, K., Gottschalk, G., 1985. Formation of d(-)-1,2-propanediol and d(-)-lactate from glucose by Clostridium sphenoides under phosphate limitation. Archives of Microbiology. 142, 87-92.

Turner, K. W., Roberton, A. M., 1979. Xylose, arabinose, and rhamnose fermentation by Bacteroides ruminicola. Appl Environ Microbiol. 38, 7-12.

Verduyn, C., Postma, E., Scheffers, W. A., Van Dijken, J. P., 1992. Effect of benzoic acid on metabolic fluxes in yeasts: a continuous-culture study on the regulation of respiration and alcoholic fermentation. Yeast. 8, 501-17.

Yang, F., Hanna, M. A., Sun, R., 2012. Value-added uses for crude glycerol--a byproduct of biodiesel production. Biotechnol Biofuels. 5, 13. 


\section{ACCEPTED MANUSCRIPT}

Yazdani, S. S., Gonzalez, R., 2007. Anaerobic fermentation of glycerol: a path to economic viability for the biofuels industry. Curr Opin Biotechnol. 18, 213-9.

Yazdani, S. S., Gonzalez, R., 2008. Engineering Escherichia coli for the efficient conversion of glycerol to ethanol and co-products. Metabolic Engineering. 10, 340-351.

Zhang, L., Tang, Y., Guo, Z., Shi, G., 2013. Engineering of the glycerol decomposition pathway and cofactor regulation in an industrial yeast improves ethanol production. J Ind Microbiol Biotechnol. 40, 1153-60.

\section{Highlights}

- Highest reported 1,2-propanediol (1,2-PDO) titer for yeast $\left(>4 \mathrm{~g} \mathrm{~L}^{-1}\right)$ from glycerol

- NADH-generating glycerol catabolic pathway crucial for 1,2-PDO formation

- Increased metabolic supply of precursor dihydroxyacetone phosphate essential

- Strong impact of medium composition on 1,2-PDO to ethanol ratio 